\title{
Facet Control of Gold Nanorods
}

\author{
Qingfeng Zhang ${ }^{\dagger}$, Lili Han ${ }^{\ddagger}$, Hao Jing ${ }^{\dagger}$, Douglas A. Blom ${ }^{\S}$, Ye Lin`, Huolin L. Xin ${ }^{\ddagger}$, and Hui Wang ${ }^{\dagger, *}$ \\ ${ }^{\dagger}$ Department of Chemistry and Biochemistry, University of South Carolina, Columbia, South Carolina \\ 29208, United States \\ $\$$ Center for Functional Nanomaterials, Brookhaven National Laboratory, Upton, New York 11973, \\ United States \\ ${ }^{\S}$ NanoCenter, University of South Carolina, Columbia, South Carolina 29208, United States \\ " Department of Chemical Engineering, University of South Carolina, Columbia, South Carolina \\ 29208, United States
}

* To whom correspondence should be addressed.

Email: wang344@mailbox.sc.edu (H.Wang); Phone: 1-803-777-2203; Fax: 1-803-777-9521. 


\begin{abstract}
While great success has been achieved in fine-tuning the aspect ratios and thereby the plasmon resonances of cylindrical Au nanorods, facet control with atomic level precision on the highly curved nanorod surfaces has long been a significantly more challenging task. The intrinsic structural complexity and lack of precise facet control of the nanorod surfaces remain the major obstacles for the atomic-level elucidation of the structure-property relationships that underpin the intriguing catalytic performance of $\mathrm{Au}$ nanorods. Here we demonstrate that the facets of single-crystalline Au nanorods can be precisely tailored using cuprous ions and cetyltrimethylammonium bromide as a unique pair of surface capping competitors to guide the particle geometry evolution during nanorod overgrowth. By deliberately maneuvering the competition between cuprous ions and cetyltrimethylammonium bromide, we have been able to create, in a highly controllable and selective manner, an entire family of nanorod-derived anisotropic multifaceted geometries whose surfaces are enclosed by specific types of well-defined high-index and low-index facets. This facet-controlled nanorod overgrowth approach also allows us to fine-tune the particle aspect ratios while well-preserving all the characteristic facets and geometric features of the faceted Au nanorods. Taking full advantage of the combined structural and plasmonic tunability, we have further studied the facet-dependent heterogeneous catalysis on wellfaceted $\mathrm{Au}$ nanorods using surface-enhanced Raman spectroscopy as an ultrasensitive spectroscopic tool with unique time-resolving and molecular finger-printing capabilities.
\end{abstract}

\footnotetext{
KEYWORDS: Gold nanorods, high-index facets, low-index facets, overgrowth, plasmon resonances, nano-catalysis, surface-enhanced Raman spectroscopy
} 
Ever since its discovery in 1990s, ${ }^{1}$ Au nanorod has been a model system for exploring the anisotropic shape evolution of nanocrystals with thermodynamically unexpected geometries. ${ }^{2-12}$ The state-of-the-art colloidal synthesis of single-crystalline $\mathrm{Au}$ nanorods involves seed-mediated anisotropic nanocrystal growth coguided by a foreign metal ion, $\mathrm{Ag}^{+}$, and halide-containing cationic surfactants, typically cetyltrimethylammonium bromide (CTAB). ${ }^{2-4,}{ }^{9-14}$ While detailed mechanistic understanding of the synergy between $\mathrm{Ag}^{+}$ions and the surfactants still remains elusive, ${ }^{15-19}$ this seedmediated growth method has become the most popular approach to the realization of precise control over both the longitudinal and transverse dimensions of cylindrical Au nanorods. Tight control over nanorod aspect ratios allows one to fine-tune the plasmon resonances over a broad spectral range that spans the entire visible and near-infrared regions. ${ }^{3,4,7,8,11}$ Such exceptional tunability of plasmondominated light absorption and scattering properties, when combined with the rich chemistry for surface functionalization of $\mathrm{Au}$, endows $\mathrm{Au}$ nanorods with great promise for applications in diverse areas, such as plasmon-enhanced spectroscopies, ${ }^{20-25}$ molecular sensing, ${ }^{26-28}$ bioimaging, ${ }^{8,} 29-33$ drug delivery, ${ }^{30,34,35}$ and photothermal cancer therapy. ${ }^{8,29,30,32,36}$

Equally important to the control over nanorod aspect ratios is the capability of fine-tailoring the surface structures of $\mathrm{Au}$ nanorods with atomic-level precision. For many biomedical and biosensing applications, the nature of the surface ligands around Au nanorods may be even more important than the $\mathrm{Au}$ core itself in terms of interfacial chemistry and biocompatibility. ${ }^{8,9,30}$ The crystallographic facets exposed on Au nanorod surfaces play pivotal roles in determining the affinity, specificity, and dynamics of the interactions between ligand molecules and Au surfaces. ${ }^{9}$ In addition, deliberate facet control opens up unique opportunities to functionalize the nanorod surfaces with desired molecular moieties in a site-selective manner, enabling the molecularly guided assembly of Au nanorods into mesoscopic hierarchical superstructures with desired architectures and functionalities. ${ }^{37-41}$ Furthermore, precise facet control is vital to the optimization of the catalytic performance of $\mathrm{Au}$ nanorods. Inhomogeneous site-specific catalytic activities were recently observed on individual single- 
crystalline Au nanorods, which are intimately tied to the geometric distribution of various local facets and defects on the nanorod surfaces. ${ }^{42}$ In striking contrast to the great success achieved in fine-tuning the aspect ratios, the seed-mediated nanorod synthesis unfortunately offers limited capability of facet

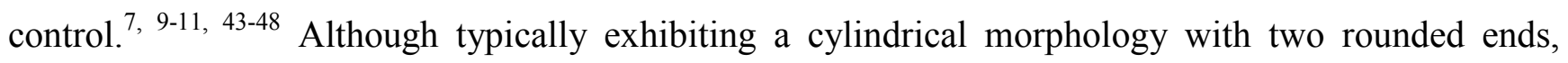
experimentally fabricated Au nanorods are essentially enclosed by multifaceted surfaces composed of a mixture of various types of high-index and low-index facets that are capped with surfactants and other adsorbates. ${ }^{9}, 43-45,49-51$ Quantitative assignment of the crystallographic facets exposed on the surfaces of $\mathrm{Au}$ nanorods, however, has long been a subject under intense debate. ${ }^{9,}$ 43-45, ${ }^{49-51}$ While catalytically active sites are abundant on the highly curved nanorod surfaces, it remains a significant challenge to quantitatively correlate the catalytic activities with the atomic-level surface structures due to the intrinsic structural complexity and poor control over the nanorod facets.

Conventional single-crystalline Au nanorods with a cylindrical morphology are typically prepared by seed-mediated growth in the presence of $\mathrm{Ag}^{+}$and CTAB. ${ }^{4-11}$ The nanorod growth is initiated by adding colloidal $\mathrm{Au}$ seeds $\left(\sim 2-4 \mathrm{~nm}\right.$ in diameter) into a growth solution containing $\mathrm{HAuCl}_{4}(\mathrm{Au}$ precursor), $\mathrm{Ag}^{+}$(structure-directing foreign ion), CTAB (surface capping surfactant), and ascorbic acid (mild reducing agent). The most convenient way to tune the nanorod aspect ratios is to vary the concentration of $\mathrm{Ag}^{+}$in the growth solution, while the aspect ratios and surface curvature of $\mathrm{Au}$ nanorods can be further fine-tuned through post-fabrication overgrowth ${ }^{46,47,52-56}$ or anisotropic oxidative etching processes. ${ }^{57,58}$ Recently, Murray and co-workers demonstrated that Au nanorods enclosed exclusively by one specific type of high-index $\{h k 0\}$ facets could be fabricated using binary surfactant mixtures instead of CTAB to guide the seed-mediated growth. ${ }^{59,60}$ These $\{h k 0\}$-faceting Au nanorods are geometrically defined as elongated tetrahexahedral (ETHH) nanoparticles (NPs). ${ }^{61,62}$ The $\{h k 0\}$ facets, composed of alternating $\{100\} /\{110\}$ terraces and steps, possess high fraction of coordinatively unsaturated surface atoms that are catalytically much more active than the close-packed surface atoms on the low-index $\{100\}$ and $\{111\}$ facets. ${ }^{63,}{ }^{64}$ More recently, we found that the $\{h k 0\}$ - 
faceting Au ETHH NPs, also known as convex nanocuboids, could be fabricated through overgrowth of preformed cylindrical $\mathrm{Au}$ nanorods in the presence of cupric $\left(\mathrm{Cu}^{2+}\right)$ ions and appropriate binary surfactant mixtures. ${ }^{65}$ In this work, we use the Au ETHH NPs as the starting materials to demonstrate that an entire family of high-index and low-index facets can be controllably created on the surfaces of single-crystalline nanorods using cuprous $\left(\mathrm{Cu}^{+}\right)$ions and $\mathrm{CTAB}$ as a unique pair of surface capping competitors to judiciously maneuver the thermodynamic and kinetic factors that govern the facet evolution during nanorod overgrowth. The unique combination of desired plasmonic properties and fine-tailored surface structures on $\mathrm{Au}$ nanorods enables us to gain detailed, quantitative insights into the facet-dependent catalytic molecular transformations on $\mathrm{Au}$ nanoparticle (NP) surfaces using surface-enhanced Raman scattering (SERS) as an in situ plasmon-enhanced spectroscopic tool.

\section{RESULTS AND DISCUSSION}

For noble metals with face centered cubic (fcc) structures, such as $\mathrm{Au}, \mathrm{Pt}$, and $\mathrm{Pd}$, the low-index $\{111\}$ and $\{100\}$ facets are thermodynamically more stable than the high-index facets and are thus highly favored during nanocrystal growth. ${ }^{63,64}$ However, the surface energies of various facets can be significantly altered when the facets interact with surfactants and/or foreign ions, allowing the nanocrystals to evolve into exotic polyhedral geometries that are enclosed by high-index facets. ${ }^{66-72}$ On the other hand, the nanocrystal facet evolution can also be kinetically controlled using appropriate combinations of structure-directing ions and surface capping surfactants to modulate the degree of supersaturation of the crystal growth units. ${ }^{73}$ Starting from the Au ETHH NPs, we found that a series of interesting nanorod-derived geometries enclosed by well-defined characteristic high-index and lowindex facets, such as elongated trisoctahedral (ETOH) NPs, concave cuboidal (CCB) NPs, quasicuboidal (QCB) NPs, truncated cuboidal (TCB) NPs, and elongated octahedral (EOH) NPs, could be 
obtained in a highly selective and controllable manner by systematically varying the molar ratio between $\mathrm{Cu}^{2+}$ and CTAB in the ETHH NP overgrowth solution.

We first used scanning electron microscopy (SEM) and transmission electron microscopy (TEM) to characterize the structural evolution of Au ETHH NPs upon their overgrowth in the presence of 14 mM CTAB and varying concentrations of $\mathrm{Cu}^{2+}$. We synthesized $\mathrm{Au}$ ETHH NPs with aspect ratio of $\sim 3$ (Figure 1A) following a previously reported seed-mediated growth method in a CTAB/oleate binary surfactant system $^{59}$ with some minor modifications. Upon exposure to an overgrowth solution containing $\mathrm{HAuCl}_{4}$, ascorbic acid (AA), and CTAB, the Au ETHH NPs evolved into ETOH NPs with significantly increased lateral dimensions (Figure 1B). The ETOH NPs represent an interesting geometry derived from elongation of a trisoctahedron enclosed by 24 high-index $\{h h k\}$ facets (composed of alternating $\{110\} /\{111\}$ terraces and steps). When $\mathrm{Cu}^{2+}$ ions were introduced into the nanorod overgrowth solution, the ETHH NPs underwent drastically different structural evolution processes and the $\mathrm{Cu}^{2+} / \mathrm{CTAB}$ molar ratio was found to be a key knob that one could adjust to finecontrol the facets of the resulting Au nanorods. At relatively low $\mathrm{Cu}^{2+}$ concentrations $\left(e . g\right.$., $\left[\mathrm{Cu}^{2+}\right]=5$ $\mu \mathrm{M})$, Au CCB NPs with well-defined concave facets were obtained (Figure 1C). As discussed in greater detail later on, each CCB NP is exclusively enclosed by 24 high-index $\{h k k\}$ facets, which are geometrically derived through combination of alternating $\{100\} /\{111\}$ terraces and steps. As the $\mathrm{Cu}^{2+}$ concentration increased, the degree of surface indentation of the CCB NPs gradually decreased until $\mathrm{Au}$ QCB NPs (Figure 1D) whose surfaces were dominated by $\{100\}$ facets formed when the concentration of $\mathrm{Cu}^{2+}$ reached $70 \mu \mathrm{M}$. Further increase in $\mathrm{Cu}^{2+}$ concentration resulted in corner truncation of the QCB NPs, giving rise to the formation of TCB NPs (Figure 1E). Each TCB NP is enclosed by $4\{100\}$ facets on the lateral sides, $2\{100\}$ facets at the ends, and $8\{111\}$ facets at the truncated corners. The corner truncation became progressively more significant as the $\mathrm{Cu}^{2+}$ concentration increased. The TCB NPs eventually evolved into EOH NPs with two sharp tips each of 


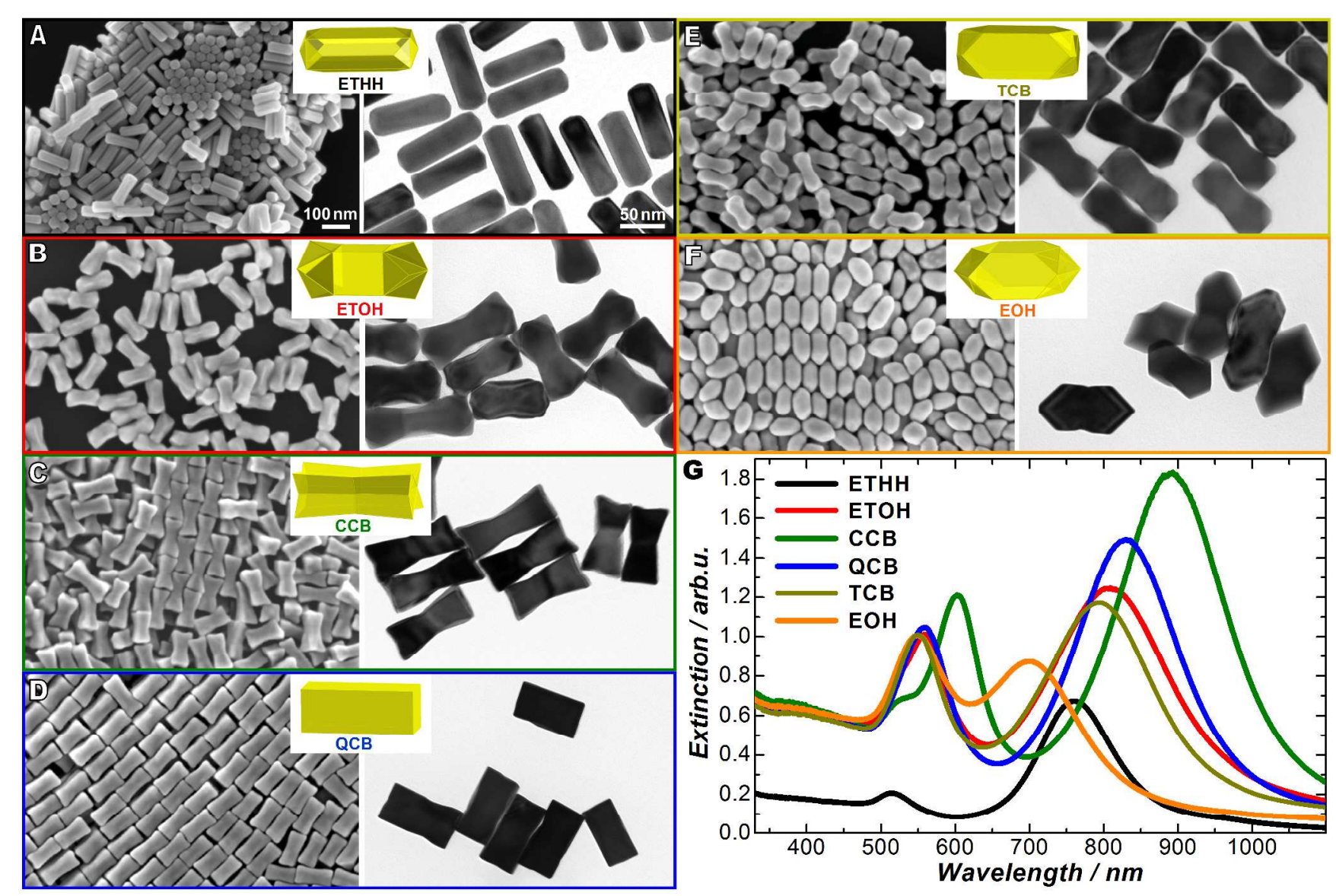

which was enclosed by $4\{111\}$ facets (Figure 1F) when the $\{100\}$ end facets completely disappeared at $\mathrm{Cu}^{2+}$ concentrations higher than $200 \mu \mathrm{M}$. The as-fabricated Au ETHH, ETOH, CCB, QCB, TCB, and EOH NPs all exhibited narrow size distributions and high geometric uniformity with yields typically higher than $95 \%$ (see low-magnification SEM images taken over large sample areas in Figure S1 in Supporting Information).

Figure 1. SEM and TEM images of Au (A) ETHH, (B) ETOH, (C) CCB, (D) QCB, (E) TCB, and (F) EOH NPs. The SEM images and TEM images are shown in the left and right columns, respectively. The insets show the geometric models of individual NPs. All the SEM and TEM images share the same scale bars in panel A. (G) Extinction spectra of colloidal Au ETHH, ETOH, CCB, QCB, TCB, and EOH NPs. The particle concentration was $\sim 1.0 \times 10^{11}$ particles $\mathrm{mL}^{-1}$ for all the samples.

Each faceted nanorod geometry exhibited its own characteristic plasmonic features in the optical extinction spectra (Figure 1G). The ETHH NPs displayed a longitudinal and a transverse plasmon resonance at $\sim 770 \mathrm{~nm}$ and $\sim 514 \mathrm{~nm}$, respectively. The longitudinal plasmon peak was much stronger 
than the transverse plasmon peak because of stronger coupling of the incident light with the plasmons along the longitudinal axis of the NPs. Upon formation of ETOH NPs, both the longitudinal and transverse plasmon resonances red-shifted and the transverse plasmon peak became significantly stronger largely due to the increased lateral dimensions of the NPs. The development of surface concavity during the transition of ETOH to CCB NPs caused significant red-shifts of both the longitudinal and transverse plasmon resonances accompanied by increase in peak intensities. Interestingly, the transverse plasmon band split into two peaks, which was a unique spectral signature of nanorods with surface indentations on their lateral sides. ${ }^{47,65}$ Decrease in surface indentation led to progressive blue-shift and weakening of both the longitudinal and transverse plasmon peaks until the formation of QCB NPs. The longitudinal plasmon peak was observed to gradually blue-shift and become weaker while the transverse plasmon peak remained very robust at essentially fixed wavelengths as the degree of corner truncation of TCB NPs increased. The transverse plasmon peak became even stronger than the longitudinal plasmon peak when the TCB NPs eventually evolved into EOH NPs. Because both the plasmon resonance wavelengths and the optical extinction spectral lineshapes were sensitively dependent on the geometric details of the faceted Au nanorods, we were able to use optical extinction spectroscopy in combination with TEM to track detailed structural evolution as the $\mathrm{Cu}^{2+} / \mathrm{CTAB}$ ratio systematically varied (Figure $\mathrm{S} 2$ in Supporting Information).

We further used high-angle annular dark-field scanning transmission electron microscopy (HAADF-STEM) to resolve the atomic-level surface structures of the faceted Au nanorods. The highresolution HAADF-STEM images shown in Figure 2 were all taken with the electron beam projected along specific zone axes of individual NPs, which allowed us to resolve the atomic structures of specific facets exposed on the nanorod surfaces. The relative orientation of each NP with respect to the electron beam was further verified by the crystalline lattices in the high-resolution HAADF-STEM images and the fast-Fourier transform patterns of the images. In Figure 2, the geometric models and atomic-level structures of various facets were also illustrated. As shown in Figure $2 \mathrm{~A}-2 \mathrm{C}$, each $\mathrm{Au}$ 
ETHH NPs was exclusively enclosed by $24\{730\}$ facets, which were identified by both the atomic steps shown in high resolution STEM images (Figure 2C-i, 2C-ii, and 2C-iii) and the characteristic dihedral angles when the particle was projected along the [001] zone axis. The $\{730\}$ facet consists of repeating high-index $\{210\}$ and $\{310\}$ local facets as illustrated in Figure 2A. The two ends of each ETOH NP were enclosed exclusively by high-index $\{221\}$ facets while the lateral side facets were indexed as $\{110\}$ (Figure 2D-2F). Four of the $24\{221\}$ facets and 2 of the $4\{110\}$ facets became parallel to the electron beam when an ETOH NP was projected along the [1 10$]$ zone axis (Figure $2 \mathrm{~F}$, 2F-i, 2F-ii, and 2F-iii). The CCB NPs, each of which was enclosed by 24 concave facets with equivalent Miller indices, exhibited orientation-dependent geometrical contours in the HAADF-STEM images (Figure 2G-J). When a CCB NP was projected along [1 $\left.1{ }^{1} 10\right]$ zone axis (Figure 2J and 2J-i), 4 of the 24 facets were aligned parallel to the electron beam, allowing us to assign the facets to high-index $\{511\}$ facet based on the arrangement of surface atoms. In comparison to the high-index faceting NPs, Au QCB NPs had a simpler geometry enclosed predominantly by 6 low-index $\{100\}$ facets with minor structural nonideality, such as slight corner truncations and defects on side facets, with respect to a perfect cuboid (Figure 2K-2L). The exposed facets on Au TCB NPs were resolved as $\{111\}$ at the truncated corners and $\{100\}$ on side and end faces (Figure S3 in Supporting Information). When a TCB NP further evolved into an EOH NP, the $\{100\}$ facets disappeared as the $4\{111\}$ facets merged at each end of the NP (Figure 2M-2O). The arrangement of surface atoms on $\{111\}$ facets were resolved by high-resolution HAADF-STEM images when an EOH NP was imaged under the project along the $\left[1^{-1} 10\right]$ zone axis (Figure 2O, 2O-i, and 2O-ii). We analyzed the characteristic angles of the high-index faceting NPs and the image intensity profiles along various lines across the NP crosssections (Figure S4 in Supporting Information), which further qualitatively confirmed the threedimensional (3D) geometric profiles of the NPs observed in the SEM and TEM images. In all the high-resolution HAADF-STEM images shown in Figure 2 and Figure S3 in Supporting Information, only the crystalline lattices of fcc Au were resolved. Neither surface deposition of metallic $\mathrm{Cu}$ nor the 
formation of $\mathrm{Au}-\mathrm{Cu}$ bimetallic alloy domains was observed, indicating the absence of metallic $\mathrm{Cu}(0)$ in these faceted nanorods.

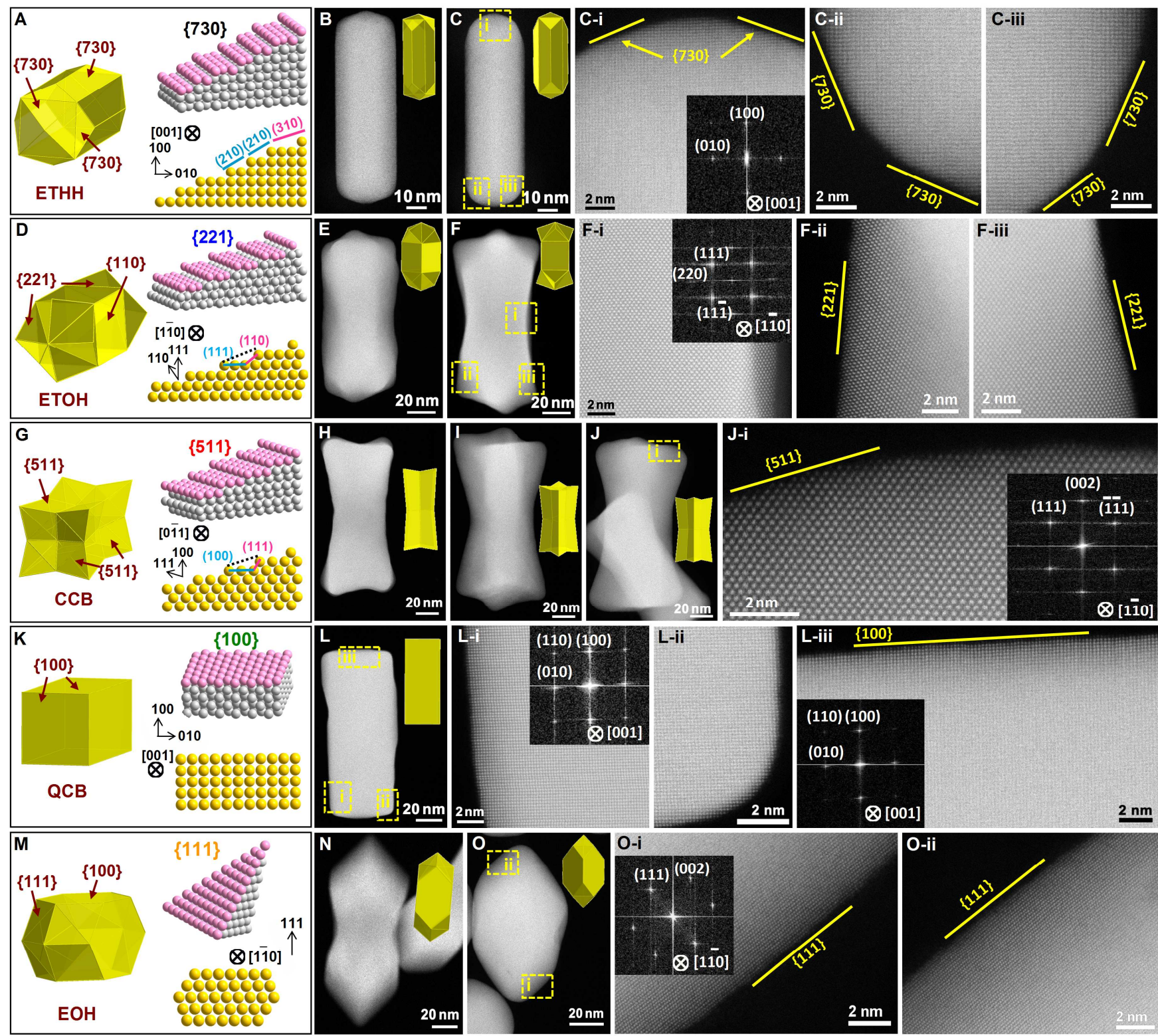

Figure 2. Atomic level surface structures of $\mathrm{Au}$ ETHH, ETOH, CCB, QCB, and EOH NPs. (A) Geometric models of an ETHH NP and $\{730\}$ facet. (B and C) HAADF-STEM images of individual ETHH NPs. (D) Geometric models of an ETOH NP and $\{221\}$ facet. (E and F) HADDF-STEM images of individual ETOH NPs. (G) Geometric models of a CCB NP and $\{511\}$ facet. (H-J) HADDF-STEM images of individual CCB NPs. (K) Geometric models of a QCB NP and $\{100\}$ facet. (L) HADDF-STEM image of an individual QCB NP. (M) Geometric models of EOH and $\{111\}$ facet. ( $\mathrm{N}$ and $\mathrm{O}$ ) HADDF-STEM images of individual EOH NPs. The insets show the geometric models of the NPs viewed at the corresponding orientation. (x-i, x-ii, x-iii, $\mathrm{x}=\mathrm{C}, \mathrm{F}, \mathrm{J}, \mathrm{L}, \mathrm{O})$ High-resolution HAADF-STEM images of various regions (i, ii, and iii) for each NP shown in panels C, F, J, L, and O, respectively. These NPs were imaged with projection along the [001] zone axis for ETHH and QCB NPs, and [1-10] zone axis for ETOH, CCB and EOH NPs. The insets in panels C-i, F-i, J-i, L-i, L-iii, and $\mathrm{O}-\mathrm{i}$ are the fast Fourier transform (FFT) patterns of the region shown in each panel, respectively. 
Complimentary to the two-dimensional (2D) microscopic imaging characterizations, electron tomography measurements allowed us to more accurately visualize the unique 3D structures and more quantitatively index the facets of the high-index faceting NPs. For the 3D electron tomography, a series of Z-contrast STEM images were acquired by tilting the specimen over a wide range of angles from $-70^{\circ}$ to $+70^{\circ}$ at every $2^{\circ}$ intervals using a field-emission instrument operated at $200 \mathrm{kV}$. The 3D tomograms were reconstructed using a multiplicative simultaneous iterative reconstruction technique (SIRT). The 3D reconstructions were visualized using isovalue surfaces in the Amira software. As shown in Figure 3, the reconstructed 3D geometries matched very well with the structural information obtained from the 2D TEM and HAADF-STEM images. The 3D tomography results further verified that the ETHH NPs and CCB NPs were predominantly enclosed by $\{730\}$ and $\{511\}$ facets, respectively. The two ends along the longitudinal axis of each ETOH NP were enclosed by $\{221\}$ facets while the lateral sides were dominated by $\{110\}$ facets. The experimentally fabricated NPs exhibited only slight deviations from the ideal geometric models. The corners and edges of the ETOH and CCB NPs appeared to be less sharp in the reconstructed 3D geometries in comparison to those observed in the TEM and SEM images possibly due to the fact that the exposure of NPs to electron beam over extended time periods during tilt-series tomography measurements may introduce slight geometric modifications to the sharp corners and edges of the NPs. In spite of their structural nonideality, all the nanorod-derived structures exhibited well-defined multifaceted geometries and their surfaces were dominated by their characteristic facets. The structural information obtained from SEM, TEM, HAADF-STEM, and 3D tomography measurements all agree with each other. 


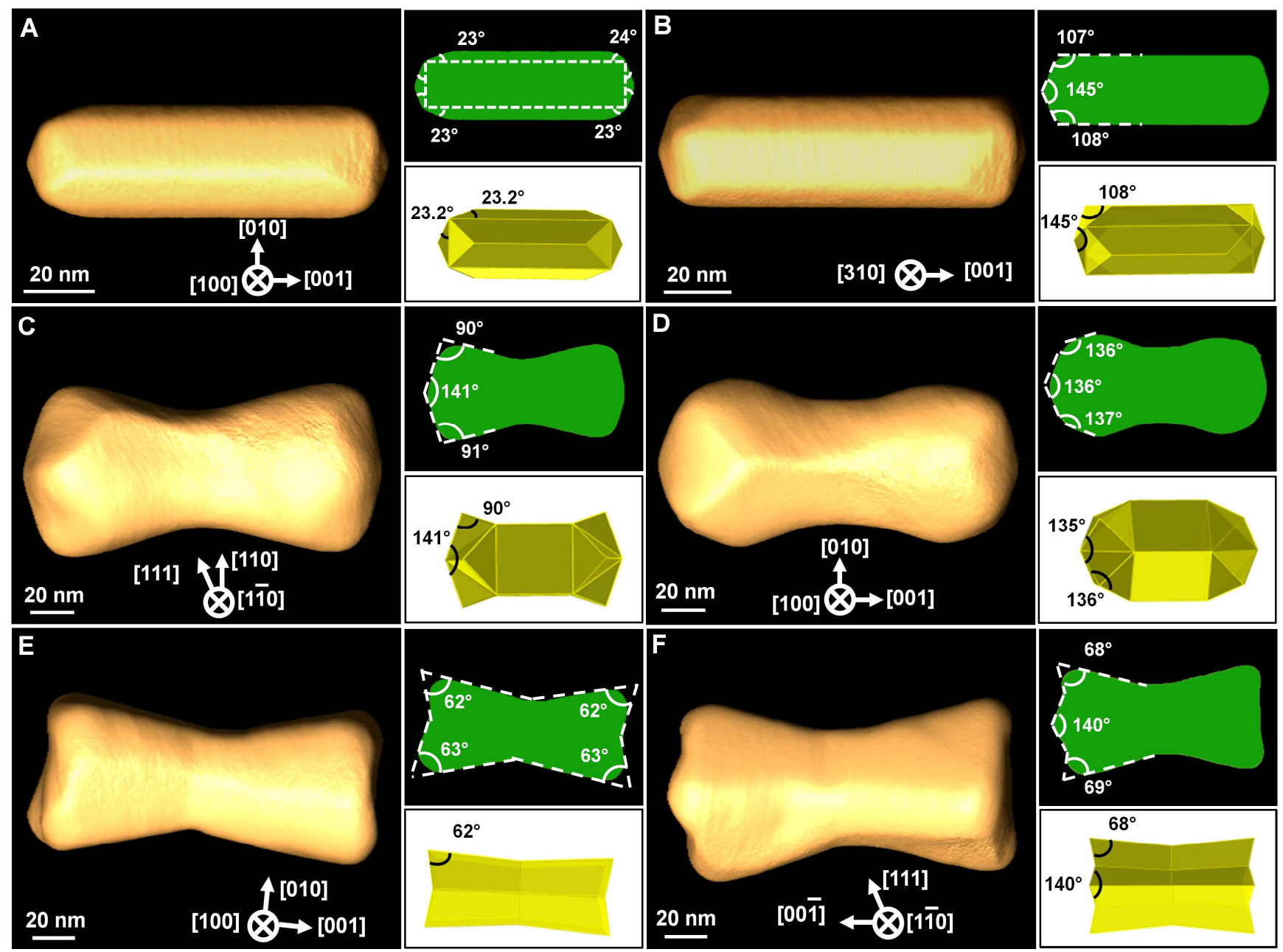

Figure 3. 3D electron tomographic reconstructions (left panels), cross-sectional views of the 2D projection of tomographic reconstructions (right upper panels), and the corresponding ideal geometric models (right bottom panels) of the high-index faceting nanorods: an ETHH NP viewed along (A) [100] zone axis and (B) [310] zone axis, an ETOH NP viewed along (C) [1-10] zone axis and (D) [100] zone axis, and a CCB NP viewed along $(\mathrm{E})$ [100] zone axis and $(\mathrm{F})[110]$ zone axis. The characteristic geometric angles on the 2D projections are labeled for each geometry.

To gain further insights into the synergistic effects of $\mathrm{Cu}^{2+}$ and $\mathrm{CTAB}$ on the facet evolution of $\mathrm{Au}$ nanorods, we used X-ray photoelectron spectroscopy (XPS) and $\zeta$-potential measurements to fully characterize the surface compositions and charges of the faceted nanorods. Energy dispersive spectroscopy (EDS) results (Figure S5 in Supporting Information) verified that the bulk composition of the faceted nanorods was monometallic $\mathrm{Au}$ and none of the $\mathrm{Cu}, \mathrm{Br}$, or $\mathrm{N}$ elements possibly existing in the surface adsorbates were detectable in EDS. However, $\mathrm{Br}$ signals were clearly resolved in XPS 
spectra collected on the Au ETHH, ETOH, CCB, QCB, TCB, and EOH NPs (Figure S6 in Supporting Information), indicating the capping of the Au surfaces with CTAB. XPS results also verified the presence of $\mathrm{Ag}$ on the surfaces of $\mathrm{Au}$ ETHH NPs (Figure S6A in Supporting Information) as a consequence of $\mathrm{Ag}^{+}$-guided seed-mediated growth. However, Ag became undetectable by XPS when the ETHH NPs overgrew into various faceted nanorods (Figure S6B-S6F in Supporting Information) because XPS was a surface characterization technique with a penetration depth of only $\sim 1 \mathrm{~nm}$ under our experimental conditions. While no XPS signal of $\mathrm{Cu}$ was detectable on the surfaces of Au ETOH NPs (Figure S6B in Supporting Information), the XPS spectral features of Cu species were clearly resolvable on the CCB, QCB, TCB, and EOH NPs (Figures S6C-S6F in Supporting Information). High-resolution XPS spectra of the $\mathrm{Cu} 2 \mathrm{p}$ region (Figure $4 \mathrm{~A}$ ) further revealed that $\mathrm{Cu}^{2+}$ was mostly reduced to $\mathrm{Cu}(\mathrm{I})$ species on the surfaces of $\mathrm{Au} \mathrm{CCB}, \mathrm{QCB}$, and EOH NPs while the XPS signals of $\mathrm{Cu}$ (II) species were almost undetectable. The relative intensities of the $\mathrm{Cu}$ (I) $2 \mathrm{p}$ XPS peaks increased in the order of $\mathrm{CCB}<\mathrm{QCB}<\mathrm{EOH}$ NPs whereas the intensities of $\mathrm{Br} 3 \mathrm{~d}$ peaks showed an opposite trend (Figure 4B), decreasing in the order of ETHH $>$ ETOH $>$ CCB $>$ QCB $>$ EOH NPs. As shown in Figure $4 \mathrm{C}$, no peak shift or split was observed in the high-resolution XPS spectra of Au 4f region in comparison to the spectrum of bulk $\mathrm{Au}$, indicating that the surface capping of $\mathrm{Au}$ facets by $\mathrm{Cu}(\mathrm{I})$ and CTAB did not modify the lattices or the electronic band structures of $\mathrm{Au}$ surface atoms to any detectable extent. This result provided additional evidence to the absence of metallic $\mathrm{Cu}$ on the overgrown faceted $\mathrm{Au}$ nanorods, which was in line with previous observations that $\mathrm{Cu}(\mathrm{II})$ ions could only be reduced to $\mathrm{Cu}(\mathrm{I})$, not metallic $\mathrm{Cu}(0)$, by ascorbic acid (AA) or sodium ascorbate in the presence of $\mathrm{CTAB} .^{70,74}$ To further verify the reduction of $\mathrm{Cu}^{2+}$ by $\mathrm{AA}$ into $\mathrm{Cu}(\mathrm{I})$ species during the nanorod overgrowth, bicinchoninic acid (BCA), a $\mathrm{Cu}^{+}$-specific chelating agent, was added into the nanorod overgrowth solution. As shown in Figure S7 in Supporting Information, the absorption peak centered at $560 \mathrm{~nm}$ in the extinction spectrum was characteristic of the water soluble, purple-colored 
$\mathrm{Cu}(\mathrm{I})$-BCA complex. ${ }^{75}$ Therefore, it was cuprous ions $\left(\mathrm{Cu}^{+}\right)$rather than cupric ions $\left(\mathrm{Cu}^{2+}\right)$ that played a key role in controlling the facet evolution of Au nanorods.
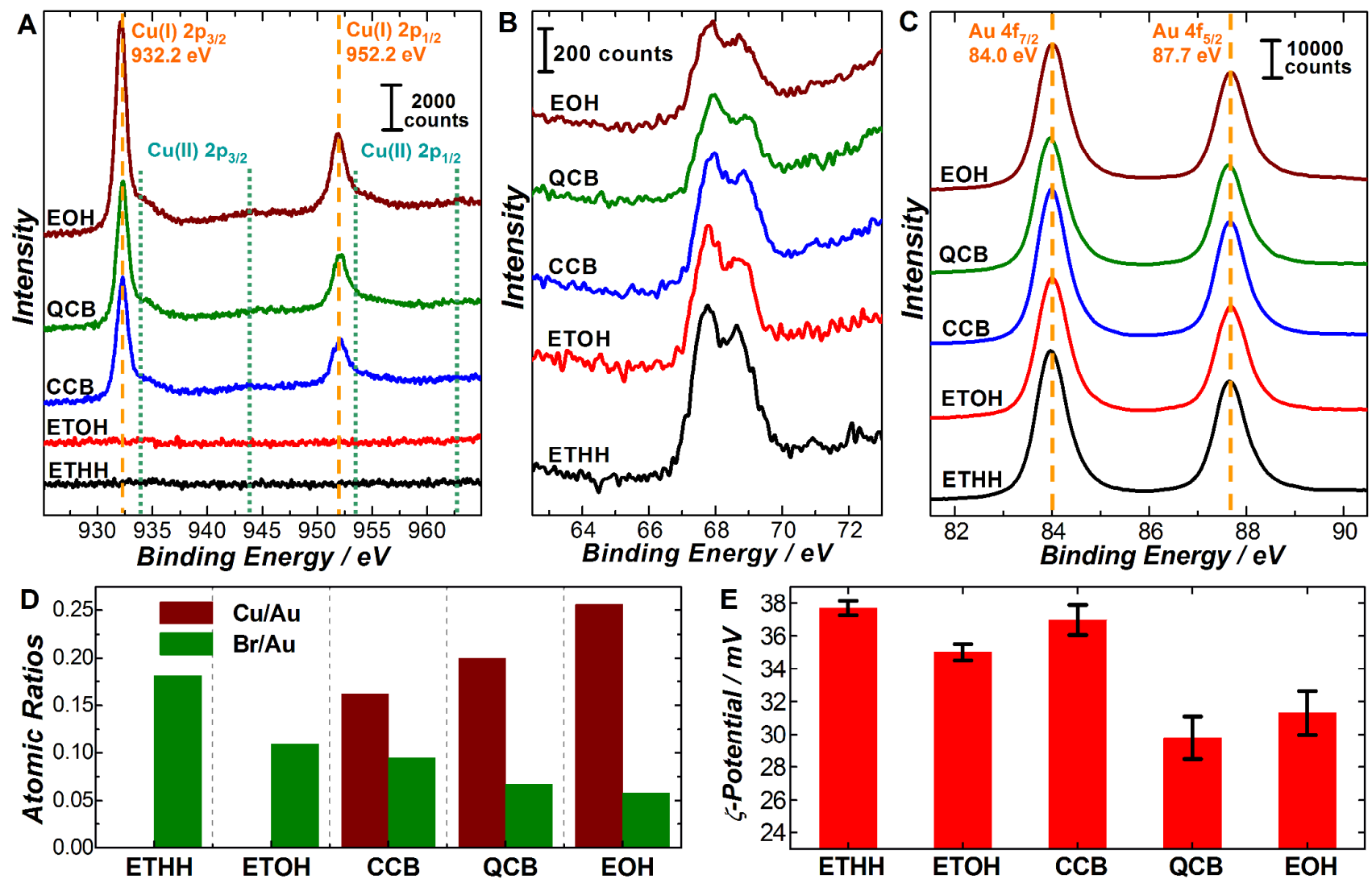

Figure 4. High-resolution XPS spectra of the (A) $\mathrm{Cu} 2 \mathrm{p}$, (B) $\mathrm{Br} 3 \mathrm{~d}$, and (C) $\mathrm{Au} 4 \mathrm{f}$ regions of $\mathrm{Au}$ ETHH, ETOH, CCB, QCB, and EOH NPs. The spectra are offset for clarity. (D) Atomic ratios of $\mathrm{Cu} / \mathrm{Au}$ and $\mathrm{Br} / \mathrm{Au}$ on the surfaces of $\mathrm{Au} \mathrm{ETHH}, \mathrm{ETOH}, \mathrm{CCB}, \mathrm{QCB}$, and $\mathrm{EOH}$ NPs probed by XPS. (E) $\zeta$-potentials of colloidal Au ETHH, ETOH, CCB, QCB, and EOH NPs. The samples for $\zeta$-potential measurements were all freshly prepared, centrifuged, and redispersed in $1 \mathrm{mM} \mathrm{CTAB}$. The concentration of the colloids for $\zeta$-potential measurements were kept at $2.0 \times 10^{11}$ particles $\mathrm{mL}^{-1}$ for all the samples. The $\mathrm{pH}$ of the colloidal suspension was 7.4.

We used the XPS results to quantify the relative packing densities of $\mathrm{Cu}$ (I) and CTAB on the NP surfaces based on the atomic ratios of $\mathrm{Cu} / \mathrm{Au}$ and $\mathrm{Br} / \mathrm{Au}$. As shown in Figure 4D, the packing densities of $\mathrm{CTAB}$ on the high-index faceting nanorods were significantly higher than those on the low-index faceting nanorods, decreasing in the order of $\mathrm{ETHH}>\mathrm{ETOH}>\mathrm{CCB}>\mathrm{QCB}>\mathrm{EOH}$ NPs. This trend correlated well with the relative thermodynamic stability of the naked facets, suggesting that high 
surface packing density of CTAB might lower the surface energies and hence stabilize the high-index facets. In contrast, $\mathrm{Cu}(\mathrm{I})$ ions, when adsorbed on the Au surfaces, appeared to favor the formation of thermodynamically stable low-index facets. As a consequence, the high-index $\{h k k\}$ facets on CCB NPs gradually evolved into $\{100\}$ facets on QCB NPs and eventually into the thermodynamically most stable $\{111\}$ facets on EOH NPs as the surface coverage of $\mathrm{Cu}(\mathrm{I})$ increased. $\mathrm{Cu}(\mathrm{I})$ ions and CTAB appeared to be a pair of surface-capping competitors because the increase in surface packing density of $\mathrm{Cu}(\mathrm{I})$ was accompanied by a decrease in packing density of CTAB (Figure 4D). The relative surface packing densities of $\mathrm{Cu}(\mathrm{I})$ and $\mathrm{CTAB}$ on the surfaces of various faceted $\mathrm{Au}$ nanorods were further correlated to the $\zeta$-potentials of the NPs (Figure 4E). All the nanostructures had positively charged surfaces due to the formation of CTAB self-assembled bilayers on Au nanorod surfaces. ${ }^{9}$ The surface adsorbed $\mathrm{Cu}(\mathrm{I})$ ions provided additional contribution to the positive surface charges of the NPs. Despite their lower surface packing density of CTAB, the CCB NPs exhibited higher surface charges than the ETOH NPs owing to the presence of surface-adsorbed $\mathrm{Cu}(\mathrm{I})$. Although $\mathrm{CTAB}$ was more sparsely packed on the surfaces of EOH NPs than QCB NPs, the EOH NPs displayed a more positive $\zeta$-potential value than the QCB NPs due to the higher surface packing density of $\mathrm{Cu}(\mathrm{I})$ ions. It is noteworthy that the $\zeta$-potential values reported here represented the apparent effective $\zeta$-potentials, which allowed us to qualitatively compare the relative surface charge densities of various NP samples. In our $\zeta$-potential measurements, a commercial zeta potentiometer was used to measure the free mobility and effective hydrodynamic sizes of NPs, which were then converted into $\zeta$ potentials using simple theoretical formulas approximating the NP as a hard sphere homogenously coated with a charged thin layer. For anisotropic Au nanorods, the obtained values of $\zeta$ potential were generally $\sim 10$ $\%$ higher than the results from the spherical approximation when theories for cylindrical particles were applied to $\zeta$ potential calculations using the actual dimensions of $\mathrm{Au}$ nanorods (determined from electron microscopy measurements). ${ }^{76}$ More quantitative determination of accurate $\zeta$-potentials, 
however, requires the incorporation of additional empirical or semi-empirical parameters, such as the geometric details of the faceted NPs and heterogeneous distribution of adsorbates on various facets, into the theoretical formulas, which is beyond the scope of this paper.

The facets of the overgrown Au nanorods could also be fine-tailored through systematic variation of CTAB concentrations while keeping $\mathrm{Cu}^{2+}$ at fixed concentrations. The morphological evolution of the faceted $\mathrm{Au}$ nanorods as the CTAB concentration varied was tracked using a combination of optical extinction spectroscopy and TEM measurements (Figure 5). At relatively low $\mathrm{Cu}^{2+}$ concentrations (e.g., $\left[\mathrm{Cu}^{2+}\right]=10 \mu \mathrm{M}$ ), a structural evolution from TCB to CCB and eventually to ETOH was observed when the $\mathrm{CTAB}$ concentration progressively increased in the range from 3 to $51 \mathrm{mM}$. At relatively high $\mathrm{Cu}^{2+}$ concentrations $\left(e . g\right.$., $\left.\left[\mathrm{Cu}^{2+}\right]=100 \mu \mathrm{M}\right)$, the NP morphologies gradually changed from EOH to TCB and eventually to CCB NPs as concentration of CTAB increased from 3 to $51 \mathrm{mM}$. Therefore, it was the molar ratio of $\mathrm{Cu}^{2+} / \mathrm{CTAB}$ rather than the absolute concentrations of $\mathrm{Cu}^{2+}$ and $\mathrm{CTAB}$ that determined the surface structures and hence the geometries of the overgrown Au nanorods. The competition between $\mathrm{Cu}^{+}$and $\mathrm{CTAB}$ could be further modulated by adding $\mathrm{BCA}$ into the overgrowth solutions. The specific and strong chelating interactions between $\mathrm{BCA}$ and $\mathrm{Cu}^{+}$ions effectively inhibited the competition between $\mathrm{Cu}^{+}$and CTAB. Therefore, Au ETOH NPs were always obtained regardless of the relative $\left[\mathrm{Cu}^{2+}\right] /[\mathrm{CTAB}]$ ratios when $\mathrm{BCA}$ was in excess with respect to $\mathrm{Cu}^{+}$(Figure $\mathrm{S} 8$ in Supporting Information). Analogous to $\mathrm{Cu}^{+}$ions, we found that $\mathrm{Pb}^{2+}$ could also compete with CTAB, allowing the ETOH NPs to evolve into CCB NPs (Figure S9 in Supporting Information). However, $\mathrm{Pb}^{2+}$ appeared to be much less effective than $\mathrm{Cu}^{+}$in terms of facet control capability. The formation of $\mathrm{CCB}$ NPs required much higher concentrations of $\mathrm{Pb}^{2+}$ than those of $\mathrm{Cu}^{2+}$ mostly likely due to the much weaker interactions of $\mathrm{Pb}^{2+}$ with $\mathrm{Au}$ surfaces compared to the $\mathrm{Cu}^{+}-\mathrm{Au}$ interactions. 

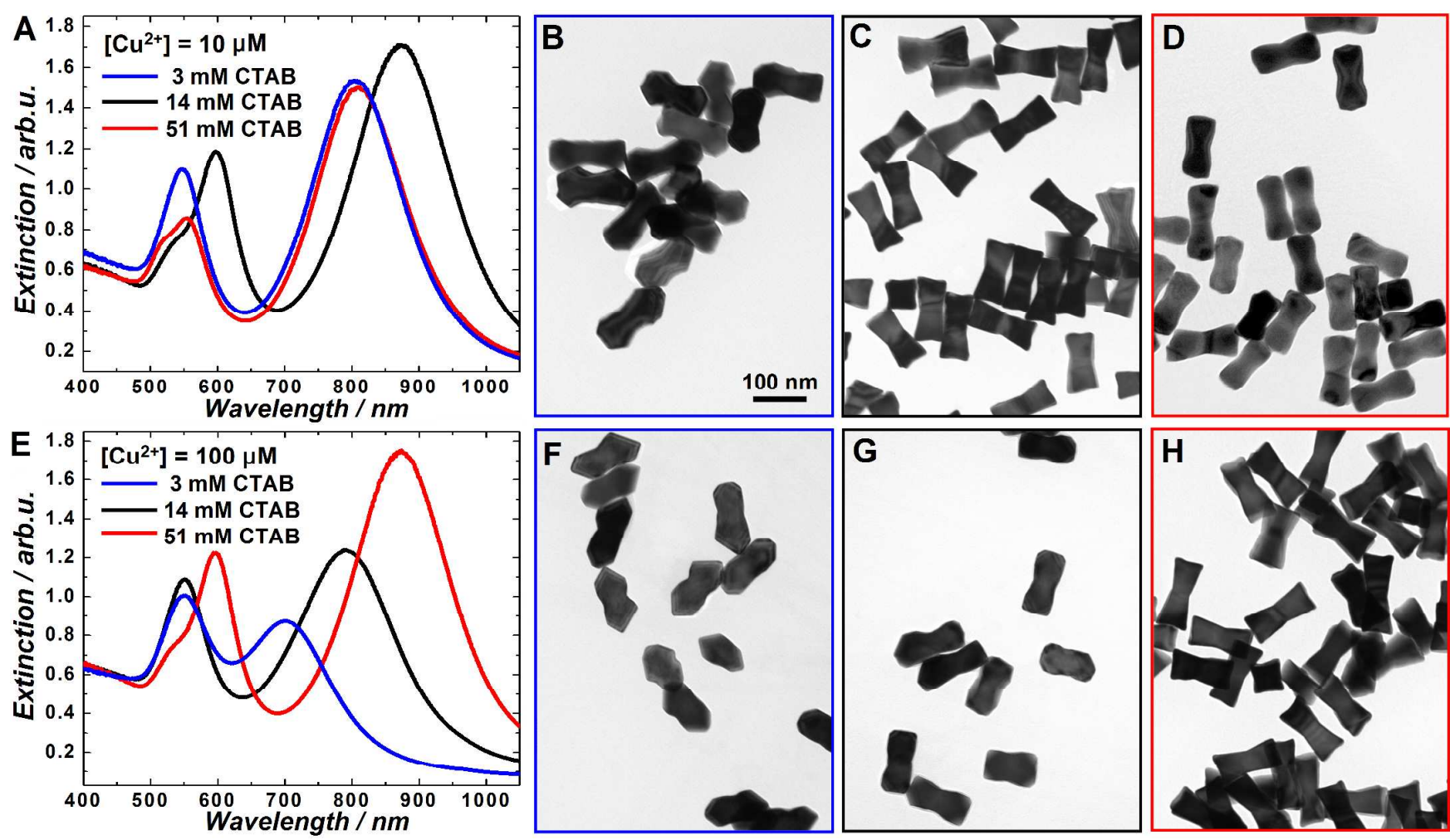

Figure 5. Extinction spectra of Au NPs obtained through overgrowth of Au ETHH NPs in the presence of various concentrations of CTAB and fixed $\mathrm{Cu}^{2+}$ concentrations: (A) $\left[\mathrm{Cu}^{2+}\right]=10 \mu \mathrm{M}$; (E) $\left[\mathrm{Cu}^{2+}\right]=$ $100 \mu \mathrm{M}$. TEM images of faceted Au nanorods obtained through overgrowth of Au ETHH NPs under various conditions: (B) $\left[\mathrm{Cu}^{2+}\right]=10 \mu \mathrm{M},[\mathrm{CTAB}]=3 \mathrm{mM}$; (C) $\left[\mathrm{Cu}^{2+}\right]=10 \mu \mathrm{M}$, [CTAB] $=14 \mathrm{mM}$; (D) $\left[\mathrm{Cu}^{2+}\right]=10 \mu \mathrm{M},[\mathrm{CTAB}]=51 \mathrm{mM} ;(\mathrm{F})\left[\mathrm{Cu}^{2+}\right]=100 \mu \mathrm{M},[\mathrm{CTAB}]=3 \mathrm{mM} ;(\mathrm{G})\left[\mathrm{Cu}^{2+}\right]=100 \mu \mathrm{M}$, $[\mathrm{CTAB}]=14 \mathrm{mM} ;(\mathrm{H})\left[\mathrm{Cu}^{2+}\right]=100 \mu \mathrm{M},[\mathrm{CTAB}]=51 \mathrm{mM}$. All TEM images share the scale bar in panel B.

Among various foreign metal ion additives, $\mathrm{Ag}^{+}$has been so far most widely used to guide the seed-mediated shape evolution of noble metal nanocrystals. $\mathrm{Ag}^{+}$ions can be used in combination with appropriate surfactants to guide the growth of a series of high-index and low-index faceting polyhedral NPs through selective surface passivation induced by underpotential deposition (UPD) of Ag. ${ }^{15,77}$ In contrast to those of $\mathrm{Ag}^{+}$, the roles of $\mathrm{Cu}^{2+}$ in seed-mediated nanocrystal growth have been much less explored. A similar mechanism involving UPD of $\mathrm{Cu}$ on $\mathrm{Au}$ seed surfaces has been proposed to interpret $\mathrm{Cu}^{2+}$-mediated shape evolution of metallic NPs. ${ }^{78,}{ }^{79}$ Although the existence of transient, localized $\mathrm{Cu}$ UPD layers on Au surfaces during nanorod overgrowth cannot be completely ruled out, the results of ex situ HAADF-STEM, XPS, and $\zeta$-potential measurements provided clear evidence on 
the absence of metallic $\mathrm{Cu}$ UPD layers on the surfaces of the overgrown Au CCB, QCB, TCB, and EOH NPs. Therefore, the facet evolution of Au nanorods observed in this work should not be simply interpreted as a consequence of selective facet passivation guided by $\mathrm{Cu}$ UPD. An alternative mechanism involved in $\mathrm{Cu}^{2+}$ ion-guided growth of $\mathrm{Au}$ nanocrystals has been recently proposed, which is based on $\mathrm{Cu}^{2+}$-catalyzed oxidative etching of $\mathrm{Au}$ surface atoms. ${ }^{56,57,80}$ The reaction occurring in this oxidative etching process can be described using the following equation:

$$
4 \mathrm{Au}^{0}+8 \mathrm{Br}^{-}+\mathrm{O}_{2}+4 \mathrm{H}^{+} \stackrel{\mathrm{Cu}^{2+}}{\longrightarrow} 4 \mathrm{AuBr}_{2}^{-}+2 \mathrm{H}_{2} \mathrm{O}
$$

This $\mathrm{Cu}^{2+}$-catalyzed oxidative etching of Au occurs in strongly acidic environments and involves the participation of bromide anions (from CTAB), the oxygen dissolved in water, protons, and $\mathrm{Cu}^{2+}$ ions. The oxidative etching of $\mathrm{Au}$ nanorods resulted in decrease of both the aspect ratios and surface curvatures of the nanorods, giving rise to spectral blue shift of the longitudinal plasmon resonances. ${ }^{57}$ When the $\mathrm{Cu}^{2+}$ ion-catalyzed oxidative etching dominated the nanorod overgrowth, the overgrowth rates greatly increased due to the etching-induced refreshment of NP surfaces, leading to the formation of thermodynamically stable $\{111\}$-faceting octahedral NPs. ${ }^{56}$ To achieve precise control over the nanorod facets, the oxidative etching of $\mathrm{Au}$ surfaces needs to be suppressed. We observed that $\mathrm{Au}$ ETHH NPs underwent a slow etching process when exposed to an aqueous solution containing $300 \mu \mathrm{M}$ $\mathrm{Cu}^{2+}, 14 \mathrm{mM} \mathrm{CTAB}$, and $0.1 \mathrm{M} \mathrm{HCl}$ under ambient air at $60{ }^{\circ} \mathrm{C}$ (Figure $\mathrm{S} 10$ in Supporting Information), which was in line with previous observations on cylindrical Au nanorods. ${ }^{57}$ This etching process was further slowed down when the temperature dropped to $30{ }^{\circ} \mathrm{C}$ (Figure S10 in Supporting Information), the temperature at which the nanorod overgrowth was carried out. In addition, we found that the presence of excessive reducing agent, AA, in the overgrowth solution suppressed the $\mathrm{Cu}^{2+}$ catalyzed oxidative etching of Au (Figure S11 in Supporting Information). Although AA is a mild reducing agent, it can effectively suppress oxidation processes, such as galvanic replacement of metallic $\mathrm{Ag}$ with $\mathrm{HAuCl}_{4}$, when it is in great excess. ${ }^{81}$ Furthermore, the $\mathrm{Cu}^{2+}$ ion-catalyzed oxidative 
etching of $\mathrm{Au}$ requires low $\mathrm{pH}$ values below 1 and therefore high concentrations $(\sim 0.1 \mathrm{M})$ of strong acids, such as $\mathrm{HCl}$ or $\mathrm{H}_{2} \mathrm{SO}_{4}$, are needed to boost the oxidative etching. ${ }^{56,57,80}$ However, under our experimental conditions for nanorod overgrowth, no additional strong acid was added and the $\mathrm{pH}$ values of the overgrowth solutions were measured to be around 4. As a consequence, no etching of $\mathrm{Au}$ ETHH NPs was observed over time period up to $24 \mathrm{~h}$ (Figure S11 in Supporting Information). While the detailed mechanistic understanding of the roles of $\mathrm{Cu}^{2+}$ and $\mathrm{CTAB}$ requires further investigations, our results provided strong evidence that the facet evolution of Au nanorods under our experimental conditions was essentially modulated by the competitive surface capping of various $\mathrm{Au}$ facets with $\mathrm{Cu}^{+}$ and $\mathrm{CTAB}$ rather than the UPD of $\mathrm{Cu}$ or $\mathrm{Cu}^{2+}$-catalyzed oxidative etching.

We further demonstrated the capability to fine-tune the particle aspect ratios using this facetcontrolled nanorod overgrowth approach (Figure S12 in Supporting Information). The aspect ratios of $\mathrm{Au}$ ETHH NPs could be fine-tuned by exposing the preformed Au ETHH NPs to the same growth solution used for the seed-mediated growth of Au ETHH NPs. The aspect ratio of Au ETHH NPs progressively decreased as the volume of the growth solution increased while the $\{730\}$ facets and the ETHH geometry were both well preserved. When the $\mathrm{Cu}^{2+} / \mathrm{CTAB}$ ratios were fixed at the optimal values for each geometry ([CTAB] was fixed at $14 \mathrm{mM}$ and $\left[\mathrm{Cu}^{2+}\right]$ were $0,5,70$, and $300 \mu \mathrm{M}$ for the ETOH, CCB, QCB, and EOH NPs, respectively), varying the amount of $\mathrm{HAuCl}_{4}$ allowed us to systematically tune the particle aspect ratios without changing the characteristic facets and morphological features of each geometry. Each faceted nanorod geometry exhibited its own characteristic aspect ratio-dependent extinction spectral features in terms of plasmon resonance wavelengths and detailed spectral line-shapes. For all the nanostructures, the transverse plasmon peaks became significantly more intense with respect to the longitudinal plasmon peaks as the particle aspect ratios decreased essentially due to the increase of the transverse dimensions of the NPs. 
A similar facet and morphological evolution process was observed upon nanorod overgrowth when $\mathrm{Ag}^{+}$ions were used instead of $\mathrm{Cu}^{2+}$ to compete with CTAB. At a fixed CTAB concentration of $14 \mathrm{mM}$, the geometry of nanorods evolved from CCB (Figure 6A) to TCB (Figure 6B) and then to EOH NPs (Figure 6C) as the $\mathrm{Ag}^{+}$concentration progressively went up. Further increase of $\mathrm{Ag}^{+}$concentration eventually led to the formation of octahedral NPs (Figure 6D). Apparently, low $\mathrm{Ag}^{+} / \mathrm{CTAB}$ ratios facilitated the formation of high-index $\{h k k\}$ facets whereas high $\mathrm{Ag}^{+} / \mathrm{CTAB}$ ratios strongly favored the formation of $\{111\}$ facets. Analogous to $\mathrm{Cu}^{+}$ions, $\mathrm{Ag}^{+}$ions served as a surface capping competitor to CTAB in guiding the facet evolution of nanorods. However, the chemistry involved in the $\mathrm{Ag}^{+}-$ mediated nanorod overgrowth was found to be strikingly different from that of the $\mathrm{Cu}^{2+}$-mediated overgrowth. During the $\mathrm{Ag}^{+}$-mediated nanorod overgrowth, $\mathrm{Ag}^{+}$was reduced to metallic $\mathrm{Ag}$ by $\mathrm{AA}$ and thus co-deposition of Ag and Au occurred on the surfaces of the Au nanorod cores (Figure 6E). In comparison to $\mathrm{Cu}, \mathrm{Ag}$ has a reduction potential much closer to that of $\mathrm{Au}$, enabling the co-deposition of $\mathrm{Ag}$ and $\mathrm{Au}$ in the presence of $\mathrm{AA}$ and $\mathrm{CTAB}$. In addition, the lattice mismatch between $\mathrm{Ag}$ and $\mathrm{Au}$ $(0.34 \%)$ is much smaller than that between $\mathrm{Cu}$ and $\mathrm{Au}(11.4 \%)$, which favors the atomic interdiffusion and thus the formation of bimetallic alloy structures. The co-reduction of Ag and $\mathrm{Au}$ under various conditions has been previously demonstrated to be a robust approach to the formation of bimetallic alloy NPs. ${ }^{82-84}$ Under our experimental conditions, the atomic ratios of Ag/Au were found to increase with the concentration of $\mathrm{Ag}^{+}$in the overgrowth solutions (Figure 6F). As shown in Figure $6 \mathrm{G}$, the structural evolution of the faceted nanorods introduced interesting modifications to the extinction spectral features. The surface concavity caused significantly red-shifts of both the longitudinal and transverse plasmon resonances while the development of corner truncation blueshifted and weakened the longitudinal plasmon peak, a similar trend as that observed in the cuprous ion-guided facet evolution. 


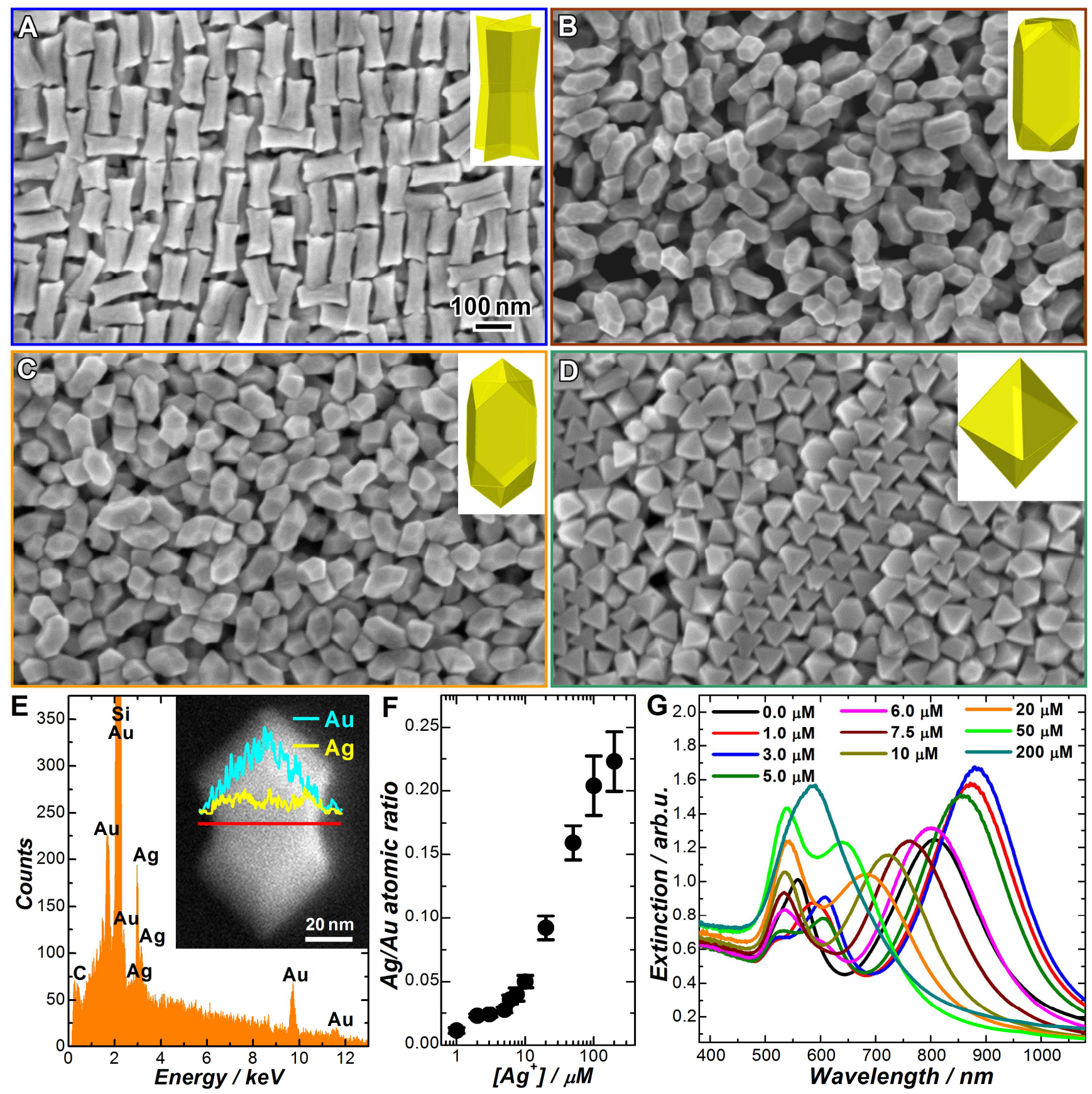

Figure 6. SEM images of NPs obtained through overgrowth of Au ETHH NPs in the presence of 14 mM CTAB and various concentrations of $\mathrm{Ag}^{+}$at: (A) $3 \mu \mathrm{M}$; (B) $7.5 \mu \mathrm{M}$; (C) $20 \mu \mathrm{M}$; (D) $200 \mu \mathrm{M}$. The inset in each panel shows the geometric model for the NPs. All SEM images share the scale bar in panel A. (E) EDS spectrum of the NPs obtained in the presence of $20 \mu \mathrm{M} \mathrm{Ag}^{+}$. The inset shows the line-scan profile of elemental distribution overlapped with the SEM image of an individual NP. (F) Atomic ratio of $\mathrm{Ag} / \mathrm{Au}$ obtained from EDS measurements as a function of the concentration of $\mathrm{Ag}^{+}$in the overgrowth solution. (G) Experimental extinction spectra of colloidal Au NPs obtained through overgrowth of $\mathrm{Au}$ ETHH NPs in the presence of various $\mathrm{Ag}^{+}$concentrations as labeled in the figure. 
Despite more than a decade of intensive investigation on the $\mathrm{Ag}^{+}$-assisted nanorod synthesis, the detailed roles of $\mathrm{Ag}^{+}$in guiding the anisotropic growth of $\mathrm{Au}$ nanorods still remain controversial. ${ }^{15-18}$ Three plausible mechanisms ${ }^{4,9,18,19}$ have been proposed regarding the roles of $\mathrm{Ag}^{+}$in controlling the nanorod aspect ratios: (1) the UPD of a submonolayer quantity of metallic Ag on the longitudinal faces of $\mathrm{Au}$ nanorods; (2) the action of a CTAB- $\mathrm{Ag}^{+}$complex as a facet-specific capping agent; and (3) the $\mathrm{Ag}^{+}$- and $\mathrm{Br}^{-}$-guided formation of rod-shaped CTAB micelles, which serve as a soft-template. Characterizing the locations of trace amount of $\mathrm{Ag}$ on the $\mathrm{Au}$ nanorod surfaces constitute the major challenge associated with the mechanistic studies. Recent studies using combined electron microscopy and advanced EDS revealed that the surface deposition of Ag exhibited no preference for a specific facet or axis of the $\mathrm{Au}$ nanorods while the dogbone-like nanostructures developed from nanorod overgrowth showed preferential Ag deposition on the ends and in the crevices. ${ }^{18}$ Although further investigations are needed to fully elucidate the synergistic effects of $\mathrm{Ag}^{+}$and CTAB on the nanorod facet evolution, our results clearly show that the competitive surface capping of nanorods with $\mathrm{Ag}^{+}$and CTAB provides a unique way to fine-tailor the facets of anisotropic Au-Ag bimetallic NPs.

The combination of fine-tailored surface structures and tunable plasmonic properties on the faceted $\mathrm{Au}$ nanorods provided a unique opportunity for us to quantitatively study the facet dependence of heterogeneous catalysis on Au nanorods using SERS as a time-resolved spectroscopic tool. As recently demonstrated by our group ${ }^{62,85,86}$ and several other groups, ${ }^{87-93}$ using SERS to characterize the interfacial molecular transformations during heterogeneous catalysis has several unique advantages, such as real-time monitoring, non-invasive detection, high sensitivity, and detailed molecular fingerprinting capability. Here we focused on the catalytic hydrogenation of surface-adsorbed 4nitrothiophenol (4-NTP) by ammonia borane (AB) as a model reaction to gain quantitative insights on the relationship between atomic surface structures and intrinsic catalytic activities of various Au facets. It has been shown that aromatic thiolated ligands, such as 4-NTP, can displace the halide-containing cationic surfactants and other physisorbed species on Au nanoparticle surfaces. ${ }^{94,95}$ The as-fabricated 
$\mathrm{Au}$ ETHH, ETOH, CCB, QCB, and EOH NPs first underwent a ligand exchange process through which self-assembled monolayers (SAMs) of 4-NTP were immobilized on the NP surfaces via the Authiol interactions to displace the surface-adsorbed $\mathrm{CTAB}$ and $\mathrm{Cu}(\mathrm{I})$ ions. The 4-NTP-coated NPs were then redispersed in water and the characteristic facets and geometric features of the NPs were both well-preserved after the ligand exchange process (Figure S13 in Supporting Information). We collected SERS spectra on colloidal NPs coated with 4-NTP SAMs at $785 \mathrm{~nm}$ excitation. Although the 4-NTP molecules were distributed over the entire NP surfaces, the overall SERS signals were dominated by the signals from the molecules adsorbed on the end facets with negligible contribution from the molecules on the lateral side facets. This is because at $785 \mathrm{~nm}$, the longitudinal plasmon resonances were more effectively excited and the field enhancements were much higher at the ends than on the lateral sides of the nanorods..$^{20,22,24,65}$ We estimated the average Raman enhancement factors (EFs) of surface-adsorbed 4-NTP by comparing the SERS signals to normal Raman signals of 4-NTP based on the Raman mode at $1338 \mathrm{~cm}^{-1}$ (see details in Supporting Information). The estimated Raman EFs were on the order of $10^{6}$ for the various faceted nanorod geometries, approaching $10^{7}$ for Au ETHH NPs (Figure S14 in Supporting Information).

The catalytic hydrogenation reactions were initiated upon exposure of the 4-NTP-coated NPs to 2 $\mathrm{mM} \mathrm{AB}$ in $1 \mathrm{mM} \mathrm{K} \mathrm{CO}_{3}$ solution at room temperature. The metallic NPs efficiently catalyzed the dehydrogenation of surface-adsorbed $\mathrm{AB}$ to generate active hydrogen, ${ }^{96}$ which then drove the hydrogenation of surface-adsorbed 4-NTP. ${ }^{65}$ We used a confocal Raman microscope with a laser focal plane $\sim 2 \mu \mathrm{m} \times 2 \mu \mathrm{m}$ in size and an effective excitation volume of $\sim 1.0 \times 10^{-16} \mathrm{~m}^{3}$ to collect the SERS spectra. Exposure of each diffusing colloidal NP to the excitation laser at relatively low power (10 $\mathrm{mW}$ ) for short time periods (limited by the diffusion time) allowed us to effectively suppress plasmondriven photoreduction of 4-NTP ${ }^{97}$ (Figure S15 in Supporting Information) and the perturbation of reaction kinetics caused by photothermal effects. ${ }^{62,85,86}$ As schematically illustrated in Figure $7 \mathrm{~A}$, this catalytic hydrogenation reaction essentially involved two key steps. 4-NTP and 4-aminothiolphenol (4- 
ATP) were the reactant and final product, respectively, and 4,4'-dimercaptoazobenzene (DMAB) was identified as the transient intermediate. The detailed assignments of the peaks in SERS spectra ${ }^{85,87,97,}$ ${ }^{98}$ were listed in Table S1 in Supporting Information. Time-resolved SERS results (Figure 7B and 6C and Figure S16 in Supporting Information) clearly showed that the reaction rates decreased in the order of $\mathrm{ETHH}>\mathrm{CCB}>\mathrm{ETOH}>\mathrm{QCB}>\mathrm{EOH}$ NPs. It is worth mentioning that all the faceted nanorod structures were enclosed by facets larger than $5 \mathrm{~nm}$ in size. Therefore, the relative reaction rates wellreflected the characteristic catalytic activities of various facets because the edge and corner atoms at the boundaries between facets only accounted for negligibly small fractions of the total surface atoms. $^{99,100}$

We used the Raman modes at $1338 \mathrm{~cm}^{-1}$ (the $\mathrm{N}-\mathrm{O}$ stretching mode of 4-NTP), $1590 \mathrm{~cm}^{-1}$ (the phenol ring mode of 4-ATP), and $1438 \mathrm{~cm}^{-1}$ (the N-N stretching mode of DMAB) to quantify the fractions of reactant $\left(\theta_{R}\right)$, product $\left(\theta_{P}\right)$, and intermediate $\left(\theta_{I}\right)$, respectively, at a function of reaction time $(t)$. To obtain $\theta_{R}(t)$ values, the peak intensities of the $1338 \mathrm{~cm}^{-1}$ mode at particular time spots, $I(t)$, were normalized against the initial peak intensities before the reactions started, $I(t=0 \mathrm{~s})$. To obtain $\theta_{P}(t)$ values, the peak intensities of the $1590 \mathrm{~cm}^{-1}$ mode at particular time spots were normalized against the peak intensities after the reaction was completed, $I(t=\infty)$. This catalytic reaction followed pseudo-first-order kinetics because AB was in great excess with respected to the surface-adsorbed 4NTP. The pseudo-first-order rate constants for the first step, $k_{l}$, were first obtained by performing least-squares curve fitting to the $\theta_{R}(t)$ trajectories $(6 \mathrm{D})$ using the following rate equation:

$$
\theta_{R}=e^{-k_{l} \times t}
$$

The pseudo-first-order rate constants for the second step, $k_{2}$, were then obtained by fitting the $\theta_{p}(t)$ trajectories using the following rate equation:

$$
\theta_{P}=1+\frac{\left(k_{1} \times e^{-k_{2} \times t}-k_{2} \times e^{-k_{1} \times t}\right)}{k_{2}-k_{1}}
$$

$\theta_{I}(t)$ was finally calculated based on the curve-fitting results using the following equation: 


$$
\theta_{I}=1-\theta_{R}-\theta_{P}
$$

The experimentally measured $\theta_{I}(t)$ were plotted after normalization of the maximum peak intensities of the $1438 \mathrm{~cm}^{-1}$ mode against the maximum $\theta_{I}$ values of the curve-fitting results.
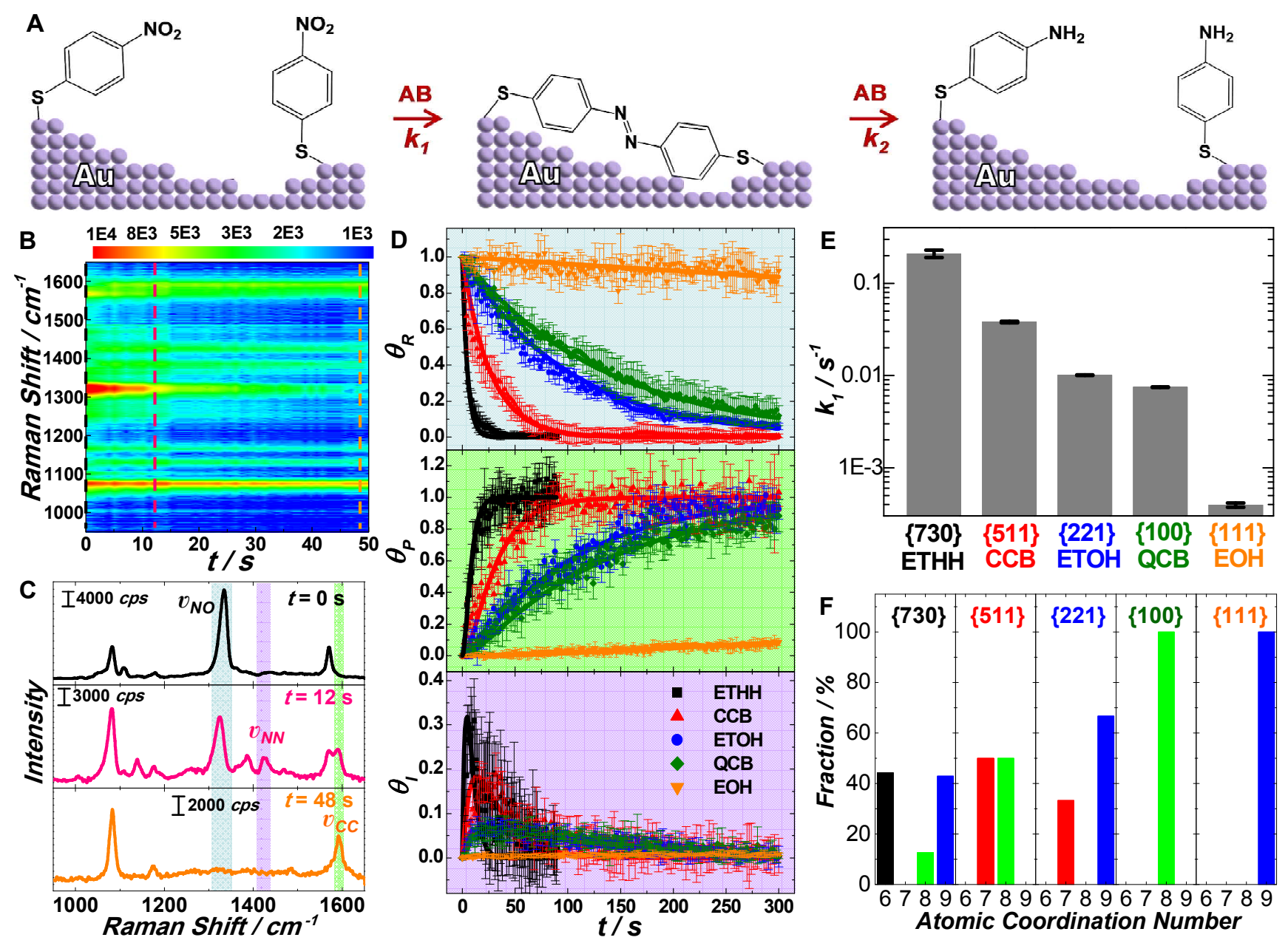

Figure 7. (A) Schematic illustration of the two-step hydrogenation process. (B) Two-dimensional colored code intensity map of time-resolved SERS spectra collected from 4-NTP molecules adsorbed on the surfaces of $\mathrm{Au}$ ETHH NPs at different reaction times upon exposure to $2 \mathrm{mM} \mathrm{AB}$. (C) Representative SERS spectra collected at reaction times of 0,12 , and $48 \mathrm{~s}$. (D) Fraction of reactant $\left(\theta_{R}\right)$ (top panel), product $\left(\theta_{P}\right)$ (middle panel), and intermediate $\left(\theta_{I}\right)$ (bottom panel) as a function of reaction time $(t)$ during the reactions catalyzed by Au ETHH, CCB, ETOH, QCB, and EOH NPs. The error bars show the standard deviations obtained from 5 experimental runs. The results of the leastsquares fitting are shown as solid curves. (E) $k_{l}$ values on Au ETHH, CCB, ETOH, QCB, and EOH NPs. (F) Fraction of surface atoms with various atomic coordination numbers for $\{730\},\{511\},\{221\}$, $\{100\}$, and $\{111\}$ facets. 
As shown in Figure 7D and 7E, the three types of high-index facets were catalytically much more active than the low-index facets toward the catalytic hydrogenation of 4-NTP. The Au ETHH NPs enclosed by $\{730\}$ facets exhibited the highest catalytic activities amongst the nanostructures investigated in this work, with $k_{1}$ approximately 500 times larger than that of the EOH NPs enclosed by the least active $\{111\}$ facets (Figure 7E). On the EOH NPs, it took more than 90 minutes for the reaction to go to completion under the current experimental conditions (Figure S17 in Supporting Information). While both $k_{1}$ and $k_{2}$ were observed to be facet dependent (Table S2 in Supporting Information), decreasing in the order of $\{730\}>\{511\}>\{221\}>\{100\}>\{111\}, k_{1}$ was much more sensitively dependent upon the facets than $k_{2}$. Interestingly, the $k_{2}$ values were always larger than $k_{1}$ regardless of nanorod geometries, suggesting that the rate-limiting step was the conversion of 4-NTP to $\mathrm{DMAB}$ and the overall reaction kinetics were essentially determined by $k_{l}$ rather than $k_{2}$. The ratios of $k_{1} / k_{2}$, which were also facet-dependent, determined both the maximum fractions of DMAB, $\theta_{I, \max }$, and the reaction time at which the $\theta_{I, \max }$ was achieved, $t_{\max }$. As shown in the bottom panel of Figure 7D, $\theta_{I}$, max decreased while $t$ max increased as $k_{1} / k_{2}$ went down. For ETOH, QCB, and EOH NPs, the fraction of DMAB $\left(\theta_{I}\right)$ remained very low throughout the entire reaction processes and the rates of product formation were dominated essentially only by $k_{1}$ (Figure S18 in Supporting Information) because $k_{2}$ was far greater than $k_{1}\left(k_{2}>10 k_{l}\right)$. As a consequence, the overall kinetics could be further simplified as a one-step first order reaction and the $\theta_{P}$ trajectories could be well described by a single-exponential function:

$$
\theta_{P}=1-e^{-k_{1} \times t}
$$

The observed facet-dependent catalytic activities correlated well with the characteristic distributions of undercoordinated surface atoms on various facets (Figure 7F). The surface atoms on the low-index $\{100\}$ and $\{111\}$ facets are close-packed with coordination numbers of 8 and 9 , respectively, and are thus catalytically less active than the high-index facets. The $\{730\}$ facet possess $44 \%$ of its surface 
atoms with coordination number of 6 , which served as highly active sites for the catalytic hydrogenation reaction. The $\{511\}$ and $\{221\}$ facets possess $50 \%$ and $33 \%$ undercoordinated surface atoms, respectively, with a coordination number of 7 . The $\{511\}$ facets were more active than the $\{221\}$ facets largely due to the higher fraction of surface atoms with coordination number of 7 . Our results provided clear experimental evidence on the crucial roles of undercoordinated surface atoms in Au-based heterogeneous catalysis, which were originally proposed based on the results obtained from CO oxidation catalyzed by oxide-supported sub-5 nm Au NPs. ${ }^{99}, 100$ The SERS-based kinetic measurements on $\mathrm{Au}$ nanorods enclosed by well-defined facets allowed us to quantitatively correlate the intrinsic activities with the atomic-level surface structures of $\mathrm{Au}$ nanocatalysts with no complication from the synergy between the Au NPs and the high surface-area oxide supports.

\section{CONCLUSIONS}

We have developed a highly robust and versatile facet-controlled nanorod overgrowth approach with unique capabilities to selectively create an entire family of well-defined facets on the surfaces of single-crystalline Au nanorods, including high-index $\{h k 0\}$ facets on ETHH NPs, high-index $\{h h k\}$ facets on ETOH NPs, high-index $\{h k k\}$ facets on CCB NPs, low-index $\{100\}$ facets on QCB NPs, and low-index $\{111\}$ facets on EOH NPs. Our success in precise facet control of Au nanorods essentially relies on the utilization of cuprous ions and $\mathrm{CTAB}$ as a unique pair of surface capping competitors to fine-control the facet evolution during nanorod overgrowth. This approach also allows for the finetuning of the particle aspect ratios while still retaining the characteristic surface structures and morphological features of each nanorod geometry. This work represents a significant advancement in nanorod synthesis and provides new mechanistic insights on the roles of foreign ions and surfacecapping surfactants in guiding the facet evolution of anisotropic nanocrystals, thereby promoting the geometry control of anisotropic nanostructures toward an unprecedented level of precision and 
versatility. The faceted Au nanorods, which exhibit fine-tailored atomic level surface structures while still inheriting the plasmonic tunability of the conventional cylindrical Au nanorods, serve as a unique multifunctional nanomaterials system that allows us to quantitatively correlate the intrinsic catalytic activities with the atomic-level surface structures of Au nanocatalysts using SERS as a time-resolved plasmon-enhanced spectroscopic tool.

\section{METHODS}

Chemicals and Materials. The chemicals and materials used in this work were listed in detail in the Supporting Information. All reagents were used as received without further purification. Ultrapure water (18.2 M $\Omega$ resistivity, Barnstead EasyPure II 7138) was used for all experiments.

Synthesis of Au ETHH NPs. Au ETHH NPs were prepared following a previously published protocol $^{59}$ with minor modifications. Briefly, colloidal Au seeds were prepared by the reducing $\mathrm{HAuCl}_{4}$ with $\mathrm{NaBH}_{4}$ in the presence of CTAB. First, $5.0 \mathrm{~mL}$ of $0.5 \mathrm{mM} \mathrm{HAuCl}_{4}$ was mixed with $5 \mathrm{~mL}$ of $0.2 \mathrm{M} \mathrm{CTAB}$ solution. Then, $1.0 \mathrm{~mL}$ of ice-cold, freshly prepared $6 \mathrm{mM} \mathrm{NaBH}_{4}$ was quickly injected into the mixture under magnetic stirring $(1200 \mathrm{rpm})$. The seed solution was stirred for $2 \mathrm{~min}$ and then left undisturbed for $30 \mathrm{~min}$ before use. To prepare the Au ETHH NP growth solution, $7.0 \mathrm{~g}$ of CTAB and $1.234 \mathrm{~g}$ of NaOL were dissolved in $250 \mathrm{~mL}$ of water at $60{ }^{\circ} \mathrm{C}$. The solution was cooled to $30{ }^{\circ} \mathrm{C}$ and then $24 \mathrm{~mL}$ of $4 \mathrm{mM} \mathrm{AgNO}_{3}$ was added. The mixture was kept undisturbed at $30{ }^{\circ} \mathrm{C}$ for 15 min, followed by the addition of $250 \mathrm{~mL}$ of $1 \mathrm{mM} \mathrm{HAuCl}_{4}$. The solution became colorless after $90 \mathrm{~min}$ of stirring at $700 \mathrm{rpm}$ and $1.8 \mathrm{~mL} \mathrm{HCl}(37 \mathrm{wt} \%$ in water, $12.1 \mathrm{M})$ was then introduced into the mixture. After another $15 \mathrm{~min}$ of slow magnetic stir at $400 \mathrm{rpm}, 1.30 \mathrm{~mL}$ of $64 \mathrm{mM}$ ascorbic acid was added. Finally, $0.4 \mathrm{~mL}$ of seed solution was injected into the growth solution and the mixture solution was vigorously stirred for another $30 \mathrm{~s}$ and then left undisturbed at $30{ }^{\circ} \mathrm{C}$ for $12 \mathrm{~h}$. The resulting Au ETHH NPs were collected by centrifugation at $7000 \mathrm{rpm}$ for $20 \mathrm{~min}$ followed by removal of the supernatant and finally redispersed in $30 \mathrm{~mL}$ of $20 \mathrm{mM}$ CTAB. 
Synthesis of Au ETOH NPs. Au ETOH NPs were prepared via overgrowth of Au ETHH NPs in the presence of $\mathrm{HAuCl}_{4}, \mathrm{CTAB}$, and AA. In a typical procedure, $200 \mu \mathrm{L}$ of colloidal Au ETHH NPs were firstly redispersed in $100 \mu \mathrm{L} 0.10 \mathrm{M} \mathrm{CTAB}$ after being washed once with water. The growth solution was prepared by sequentially adding $\mathrm{H}_{2} \mathrm{O}(7.40 \mathrm{~mL}), \mathrm{HAuCl}_{4}(0.2 \mathrm{~mL}, 10 \mathrm{mM})$, and AA (1.0 $\mathrm{mL}, 0.10 \mathrm{M})$ into a CTAB $(1.30 \mathrm{~mL}, 0.10 \mathrm{M})$ solution. After gently mixing the growth solution for 30 $\mathrm{s}$, the growth of Au ETOH was initiated by adding $100 \mu \mathrm{L}$ of the Au ETHH NPs (in 0.1 M CTAB). The reaction solution was gently mixed for $30 \mathrm{~s}$ immediately after the addition of Au ETHH NPs and then left undisturbed at $30{ }^{\circ} \mathrm{C}$ for $1 \mathrm{~h}$. The obtained Au ETOH NPs were washed with water twice through centrifugation/redispersion cycles, and finally redispersed in $200 \mu \mathrm{L}$ of $20 \mathrm{mM} \mathrm{CTAB}$. The size and aspect-ratio of ETOH can be controlled by simply adjusting amount of $\mathrm{HAuCl}_{4}$ added. The total volume of the growth solutions was always fixed at $10.0 \mathrm{~mL}$.

Synthesis of Au CCB, QCB, TCB, and EOH NPs. Au CCB, QCB, TCB, and EOH NPs were synthesized via overgrowth of $\mathrm{Au}$ ETHH NPs in the presence of $\mathrm{Cu}^{2+}, \mathrm{HAuCl}_{4}, \mathrm{CTAB}$, and AA. In a typical procedure of CCB NP synthesis, $200 \mu \mathrm{L}$ of Au ETHH NPs were first redispersed in $100 \mu \mathrm{L}$ 0.10 M CTAB after being washed once with water. The growth solution was prepared by sequentially adding $\mathrm{H}_{2} \mathrm{O}(7.395 \mathrm{~mL}), \mathrm{HAuCl}_{4}(0.2 \mathrm{~mL}, 10 \mathrm{mM}), \mathrm{Cu}\left(\mathrm{NO}_{3}\right)_{2}(5 \mu \mathrm{L}, 10 \mathrm{mM})$, and $\mathrm{AA}(1.0 \mathrm{~mL}, 0.10$ M) into a CTAB $(1.30 \mathrm{~mL}, 0.10 \mathrm{M})$ solution. After gently mixing the growth solution for $30 \mathrm{~s}$, the growth of Au CCB NPs was initiated by adding $100 \mu \mathrm{L}$ of the Au ETHH NPs (in 0.1 M CTAB). The reaction solution was gently mixed for $30 \mathrm{~s}$ immediately after the addition of Au ETHH NPs and then left undisturbed at $30{ }^{\circ} \mathrm{C}$ for $1 \mathrm{~h}$. The obtained Au CCB NPs were washed with water twice through centrifugation/redispersion cycles, and finally redispersed in $200 \mu \mathrm{L}$ of $20 \mathrm{mM} \mathrm{CTAB}$. The morphologies of $\mathrm{Au}$ CCB NPs evolved into QCB, TCB, and EOH when increasing amount of $\mathrm{Cu}^{2+}$ was added into the growth solution. The morphologies of the NPs could be controlled by adjusting the molar ratios between $\mathrm{Cu}^{2+}$ and $\mathrm{CTAB}$. In the presence of $14 \mathrm{mM} \mathrm{CTAB}$, the optimal concentrations of 
$\mathrm{Cu}^{2+}$ were $5 \mu \mathrm{M}$ for $\mathrm{CCB}, 70 \mu \mathrm{M}$ for QCB, $100 \mu \mathrm{M}$ for TCB, and $300 \mu \mathrm{M}$ for EOH NPs, respectively. The total volume of the growth solutions was always fixed at $10.0 \mathrm{~mL}$.

Overgrowth of Au ETHH NPs in CTAB/NaOL Binary Surfactants. The ETHH morphology was well-preserved while the particle aspect ratios decreased during overgrowth of Au ETHH NPs in the presence of $\mathrm{Ag}^{+}, \mathrm{HAuCl}_{4}, \mathrm{CTAB}, \mathrm{NaOL}, \mathrm{HCl}$, and AA. More details of the overgrowth of $\mathrm{Au}$ ETHH NPs in CTAB/NaOL binary surfactants can be found in Supporting Information.

$\mathrm{Ag}^{+}$-Guided Overgrowth of Au ETHH NPs. The ETHH NPs evolved into CCB, TCB, and EOH NPs composed of Au nanorod core and Au-Ag alloy shell upon overgrowth of Au ETHH NPs in the presence of $\mathrm{Ag}^{+}, \mathrm{HAuCl}_{4}, \mathrm{CTAB}$, and AA. More experimental details of $\mathrm{Ag}^{+}$-guided overgrowth of Au ETHH NPs in CTAB are included in Supporting Information.

Characterizations. The morphologies, structures, compositions, and surface properties of the NPs were characterized by TEM, SEM, EDS, HAADF-STEM, XPS, and $\zeta$-potential measurements. The optical extinction spectra of the NPs were measured using a Beckman Coulter Du 640 spectrophotometer. Raman spectra were obtained on a Bayspec Nomadic ${ }^{T M}$ Raman microscopy built on an Olympus BX51 microscope equipped with a $785 \mathrm{~nm} \mathrm{CW}$ diode laser. SERS spectra were collected on colloidal suspensions of Au ETHH, ETOH, CCB, QCB, and EOH NPs coated with 4-NTP SAMs. Details of NP characterizations, SERS measurements, and estimation of Raman enhancement factors are presented in Supporting Information.

Catalytic Reaction Kinetics Studied by Time-Resolved SERS. To use SERS to study the catalytic reactions, we first pre-adsorbed SAMs of 4-NTP onto the surfaces of Au ETHH, ETOH, CCB, QCB, and EOH NPs. The nanoparticle-catalyzed 4-NTP hydrogenation occurred at room temperature upon the addition of $20 \mu \mathrm{L}$ of $\mathrm{Au}$ NPs $\left(\sim 1.0 \times 10^{11}\right.$ particles $\left.\mathrm{mL}^{-1}\right), 50 \mu \mathrm{L}$ of ultrapure water, $10 \mu \mathrm{L}$ of $10 \mathrm{mM} \mathrm{K}_{2} \mathrm{CO}_{3}$, and $20 \mu \mathrm{L}$ of $10 \mathrm{mM} \mathrm{AB}$ in a $0.5 \mathrm{~mL}$ Eppendorf centrifuge tube. The kinetics of the catalyzed reactions was measured in real time using time-resolved SERS. The excitation 
laser was focused on the reaction mixture using a $10 \times$ objective [Numerical Aperture $(\mathrm{NA})=0.30$, working distance $(\mathrm{WD})=11.0 \mathrm{~mm}$, Olympus MPLFLN]. The laser power was measured to be 10.0 $\mathrm{mW}$ at the samples and the signal acquisition time was $1 \mathrm{~s}$ for Au ETHH NPs, and 2s for all other samples, respectively. Successive SERS spectra were collected during the reaction until completion of the reduction of 4-NTP into 4-ATP. The total volume of the reaction mixtures was fixed at $100 \mu \mathrm{L}$.

\section{ASSOCIATED CONTENT}

Supporting Information. Additional experimental details, tables, and figures including SEM images, TEM images, optical extinction spectra, EDS results, XPS results, HAADF-STEM images, and SERS spectra as noted in the text. This material is available free of charge via the Internet at http://pubs.acs.org.

\section{AUTHOR INFORMATION}

\section{Corresponding Author}

*Email: wang344@mailbox.sc.edu (H.W.); Phone: 803-777-2203; Fax: 803-777-9521.

\section{Author Contributions}

Q.Z. and H.W. conceived the idea. Q.Z. and H.J. synthesized the nanostructures. Q.Z. did the SEM, TEM, EDS, Raman, optical extinction, and $\zeta$-potential measurements. L.H. and H.L.X. did the electron tomography measurements. D.A.B. did the high-resolution HAADF-STEM measurements. Y.L. did the XPS measurements. H.W. supervised the research. Q.Z. and H.W. wrote the paper. All authors contributed to the discussion of the results and approved the manuscript submission. 


\section{Notes}

The authors declare no competing financial interests.

\section{ACKNOWLEDGMENT}

This work was supported by a National Science Foundation CAREER Award (NSF DMR-1253231), an ASPIRE-I Track-I Award from the University of South Carolina Office of Vice President for Research, and the University of South Carolina Startup Funds. The authors thank the University of South Carolina Electron Microscopy Center and W.M. Keck Open Laboratory for instrument use and technical assistance. The electron tomography results were obtained using the electron microscopy facility of the Center for Functional Nanomaterials, which is a United States Department of Energy (DOE) Office of Science Facility, at Brookhaven National Laboratory under Contract No. DESC0012704. 


\section{REFERENCES}

1. Yu, Y. Y.; Chang, S. S.; Lee, C. L.; Wang, C. R. C. Gold Nanorods: Electrochemical Synthesis and Optical Properties. J. Phys. Chem. B 1997, 101, 6661-6664.

2. Jana, N. R.; Gearheart, L.; Murphy, C. J. Wet Chemical Synthesis of High Aspect Ratio Cylindrical Gold Nanorods. J. Phys. Chem. B 2001, 105, 4065-4067.

3. Nikoobakht, B.; El-Sayed, M. A. Preparation and Growth Mechanism of Gold Nanorods (NRs) using Seed-Mediated Growth Method. Chem. Mater. 2003, 15, 1957-1962.

4. Murphy, C. J.; San, T. K.; Gole, A. M.; Orendorff, C. J.; Gao, J. X.; Gou, L.; Hunyadi, S. E.; Li, T. Anisotropic Metal Nanoparticles: Synthesis, Assembly, and Optical Applications. J. Phys. Chem. B 2005, 109, 13857-13870.

5. Grzelczak, M.; Perez-Juste, J.; Mulvaney, P.; Liz-Marzan, L. M. Shape Control in Gold Nanoparticle Synthesis. Chem. Soc. Rev. 2008, 37, 1783-1791.

6. Sau, T. K.; Murphy, C. J. Seeded High Yield Synthesis of Short Au Nanorods in Aqueous Solution. Langmuir 2004, 20, 6414-6420.

7. Perez-Juste, J.; Pastoriza-Santos, I.; Liz-Marzan, L. M.; Mulvaney, P. Gold Nanorods: Synthesis, Characterization and Applications. Coord. Chem. Rev. 2005, 249, 1870-1901.

8. Jain, P. K.; Huang, X. H.; El-Sayed, I. H.; El-Sayed, M. A. Noble Metals on the Nanoscale: Optical and Photothermal Properties and Some Applications in Imaging, Sensing, Biology, and Medicine. Acc. Chem. Res. 2008, 41, 1578-1586.

9. Murphy, C. J.; Thompson, L. B.; Alkilany, A. M.; Sisco, P. N.; Boulos, S. P.; Sivapalan, S. T.; Yang, J. A.; Chernak, D. J.; Huang, J. Y. The Many Faces of Gold Nanorods. J. Phys. Chem. Lett. 2010, $1,2867-2875$.

10. Lohse, S. E.; Murphy, C. J. The Quest for Shape Control: A History of Gold Nanorod Synthesis. Chem. Mater. 2013, 25, 1250-1261. 
11. Chen, H. J.; Shao, L.; Li, Q.; Wang, J. F. Gold Nanorods and their Plasmonic Properties. Chem. Soc. Rev. 2013, 42, 2679-2724.

12. Johnson, C. J.; Dujardin, E.; Davis, S. A.; Murphy, C. J.; Mann, S. Growth and Form of Gold Nanorods Prepared by Seed-Mediated, Surfactant-Directed Synthesis. J. Mater. Chem. 2002, 12, $1765-$ 1770.

13. Vigderman, L.; Zubarev, E. R. High-Yield Synthesis of Gold Nanorods with Longitudinal SPR Peak Greater than $1200 \mathrm{~nm}$ Using Hydroquinone as a Reducing Agent. Chem. Mater. 2013, 25, 14501457.

14. Ye, X. C.; Jin, L. H.; Caglayan, H.; Chen, J.; Xing, G. Z.; Zheng, C.; Doan-Nguyen, V.; Kang, Y. J.; Engheta, N.; Kagan, C. R.; Murray, C. B. Improved Size-Tunable Synthesis of Monodisperse Gold Nanorods through the Use of Aromatic Additives. ACS Nano 2012, 6, 2804-2817.

15. Liu, M. Z.; Guyot-Sionnest, P. Mechanism of Silver(I)-Assisted Growth of Gold Nanorods and Bipyramids. J. Phys. Chem. B 2005, 109, 22192-22200.

16. Edgar, J. A.; McDonagh, A. M.; Cortie, M. B. Formation of Gold Nanorods by a Stochastic "Popcorn" Mechanism. ACS Nano 2012, 6, 1116-1125.

17. Walsh, M. J.; Barrow, S. J.; Tong, W. M.; Funston, A. M.; Etheridge, J. Symmetry Breaking and Silver in Gold Nanorod Growth. ACS Nano 2015, 9, 715-724.

18. Jackson, S. R.; McBride, J. R.; Rosenthal, S. J.; Wright, D. W. Where's the Silver? Imaging Trace Silver Coverage on the Surface of Gold Nanorods. J. Am. Chem. Soc. 2014, 136, 5261-5263.

19. Almora-Barrios, N.; Novell-Leruth, G.; Whiting, P.; Liz-Marzan, L. M.; Lopez, N. Theoretical Description of the Role of Halides, Silver, and Surfactants on the Structure of Gold Nanorods. Nano Lett. 2014, 14, 871-875.

20. Nikoobakht, B.; Wang, J. P.; El-Sayed, M. A. Surface-Enhanced Raman Scattering of Molecules Adsorbed on Gold Nanorods: Off-Surface Plasmon Resonance Condition. Chem. Phys. Lett. 2002, 366, 17-23. 
21. Alvarez-Puebla, R. A.; Agarwal, A.; Manna, P.; Khanal, B. P.; Aldeanueva-Potel, P.; CarboArgibay, E.; Pazos-Perez, N.; Vigderman, L.; Zubarev, E. R.; Kotov, N. A.; et al. Gold Nanorods 3DSupercrystals as Surface Enhanced Raman Scattering Spectroscopy Substrates for the Rapid Detection of Scrambled Prions. Proc. Natl. Acad. Sci. U. S. A. 2011, 108, 8157-8161.

22. Sivapalan, S. T.; DeVetter, B. M.; Yang, T. K.; van Dijk, T.; Schulmerich, M. V.; Carney, P. S.; Bhargava, R.; Murphy, C. J. Off-Resonance Surface-Enhanced Raman Spectroscopy from Gold Nanorod Suspensions as a Function of Aspect Ratio: Not What We Thought. ACS Nano 2013, 7, 20992105.

23. Abadeer, N. S.; Brennan, M. R.; Wilson, W. L.; Murphy, C. J. Distance and Plasmon Wavelength Dependent Fluorescence of Molecules Bound to Silica-Coated Gold Nanorods. ACS Nano 2014, $8,8392-8406$.

24. Fu, Y.; Zhang, J.; Lakowicz, J. R. Plasmon-Enhanced Fluorescence from Single Fluorophores End-Linked to Gold Nanorods. J. Am. Chem. Soc. 2010, 132, 5540-5541.

25. Nepal, D.; Drummy, L. F.; Biswas, S.; Park, K.; Vaia, R. A. Large Scale Solution Assembly of Quantum Dot-Gold Nanorod Architectures with Plasmon Enhanced Fluorescence. ACS Nano 2013, 7, 9064-9074.

26. Mayer, K. M.; Hafner, J. H. Localized Surface Plasmon Resonance Sensors. Chem. Rev. 2011, $111,3828-3857$.

27. Lee, K. S.; El-Sayed, M. A. Gold and Silver Nanoparticles in Sensing and Imaging: Sensitivity of Plasmon Response to Size, Shape, and Metal Composition. J. Phys. Chem. B 2006, 110, 1922019225.

28. Murphy, C. J.; Gole, A. M.; Hunyadi, S. E.; Stone, J. W.; Sisco, P. N.; Alkilany, A.; Kinard, B. E.; Hankins, P. Chemical Sensing and Imaging with Metallic Nanorods. Chem. Commun. 2008, 544557. 
29. Huang, X. H.; El-Sayed, I. H.; Qian, W.; El-Sayed, M. A. Cancer Cell Imaging and Photothermal Therapy in the Near-Infrared Region by Using Gold Nanorods. J. Am. Chem. Soc. 2006, $128,2115-2120$.

30. Huang, X. H.; Neretina, S.; El-Sayed, M. A. Gold Nanorods: From Synthesis and Properties to Biological and Biomedical Applications. Adv. Mater. 2009, 21, 4880-4910.

31. Wang, H. F.; Huff, T. B.; Zweifel, D. A.; He, W.; Low, P. S.; Wei, A.; Cheng, J. X. In vitro and in vivo Two-Photon Luminescence Imaging of Single Gold Nanorods. Proc. Natl. Acad. Sci. U. S. A. 2005, 102, 15752-15756.

32. Tong, L.; Wei, Q. S.; Wei, A.; Cheng, J. X. Gold Nanorods as Contrast Agents for Biological Imaging: Optical Properties, Surface Conjugation and Photothermal Effects. Photochem. Photobiol. 2009, 85, 21-32.

33. Ding, H.; Yong, K. T.; Roy, I.; Pudavar, H. E.; Law, W. C.; Bergey, E. J.; Prasad, P. N. Gold Nanorods Coated with Multilayer Polyelectrolyte as Contrast Agents for Multimodal Imaging. J. Phys. Chem. C 2007, 111, 12552-12557.

34. Bonoiu, A. C.; Mahajan, S. D.; Ding, H.; Roy, I.; Yong, K. T.; Kumar, R.; Hu, R.; Bergey, E. J.; Schwartz, S. A.; Prasad, P. N. Nanotechnology Approach for Drug Addiction Therapy: Gene Silencing using Delivery of Gold Nanorod-siRNA Nanoplex in Dopaminergic Neurons. Proc. Natl. Acad. Sci. U. S. A. 2009, 106, 5546-5550.

35. Wijaya, A.; Schaffer, S. B.; Pallares, I. G.; Hamad-Schifferli, K. Selective Release of Multiple DNA Oligonucleotides from Gold Nanorods. ACS Nano 2009, 3, 80-86.

36. Huang, X. H.; Jain, P. K.; El-Sayed, I. H.; El-Sayed, M. A. Gold Nanoparticles: Interesting Optical Properties and Recent Applications in Cancer Diagnostic and Therapy. Nanomedicine 2007, 2, 681-693.

37. Sun, Z. H.; Ni, W. H.; Yang, Z.; Kou, X. S.; Li, L.; Wang, J. F. pH-Controlled Reversible Assembly and Disassembly of Gold Nanorods. Small 2008, 4, 1287-1292. 
38. Liu, K.; Nie, Z. H.; Zhao, N. N.; Li, W.; Rubinstein, M.; Kumacheva, E. Step-Growth Polymerization of Inorganic Nanoparticles. Science 2010, 329, 197-200.

39. Nie, Z. H.; Fava, D.; Rubinstein, M.; Kumacheva, E. "Supramolecular" Assembly of Gold Nanorods End-Terminated with Polymer "Pom-Poms": Effect of Pom-Pom Structure on the Association Modes. J. Am. Chem. Soc. 2008, 130, 3683-3689.

40. Joseph, S. T. S.; Ipe, B. I.; Pramod, P.; Thomas, K. G. Gold Nanorods to Nanochains: Mechanistic Investigations on their Longitudinal Assembly using Alpha,Omega-Alkanedithiols and Interplasmon Coupling. J. Phys. Chem. B 2006, 110, 150-157.

41. Nie, Z. H.; Fava, D.; Kumacheva, E.; Zou, S.; Walker, G. C.; Rubinstein, M. Self-Assembly of Metal-Polymer Analogues of Amphiphilic Triblock Copolymers. Nat. Mater. 2007, 6, 609-614.

42. Zhou, X. C.; Andoy, N. M.; Liu, G. K.; Choudhary, E.; Han, K. S.; Shen, H.; Chen, P. Quantitative Super-Resolution Imaging Uncovers Reactivity Patterns on Single Nanocatalysts. Nat. Nanotechnol. 2012, 7, 237-241.

43. Katz-Boon, H.; Rossouw, C. J.; Weyland, M.; Funston, A. M.; Mulvaney, P.; Etheridge, J. Three-Dimensional Morphology and Crystallography of Gold Nanorods. Nano Lett. 2011, 11, 273 278.

44. Carbo-Argibay, E.; Rodriguez-Gonzalez, B.; Gomez-Grana, S.; Guerrero-Martinez, A.; Pastoriza-Santos, I.; Perez-Juste, J.; Liz-Marzan, L. M. The Crystalline Structure of Gold Nanorods Revisited: Evidence for Higher-Index Lateral Facets. Angew. Chem. Int. Edit. 2010, 49, 9397-9400.

45. Gai, P. L.; Harmer, M. A. Surface Atomic Defect Structures and Growth of Gold Nanorods. Nano Lett. 2002, 2, 771-774.

46. Sohn, K.; Kim, F.; Pradel, K. C.; Wu, J. S.; Peng, Y.; Zhou, F. M.; Huang, J. X. Construction of Evolutionary Tree for Morphological Engineering of Nanoparticles. ACS Nano 2009, 3, 2191-2198.

47. Huang, Y. J.; Wu, L.; Chen, X. D.; Bai, P.; Kim, D. H. Synthesis of Anisotropic Concave Gold Nanocuboids with Distinctive Plasmonic Properties. Chem. Mater. 2013, 25, 2470-2475. 
48. Zhang, L. F.; Zhang, C. Y. Controlled Growth of Concave Gold Nanobars with High SurfaceEnhanced Raman-Scattering and Excellent Catalytic Activities. Nanoscale 2013, 5, 5794-5800.

49. Goris, B.; Bals, S.; Van den Broek, W.; Carbo-Argibay, E.; Gomez-Grana, S.; Liz-Marzan, L. M.; Van Tendeloo, G. Atomic-Scale Determination of Surface Facets in Gold Nanorods. Nat. Mater. 2012, $11,930-935$.

50. Wang, Z. L.; Gao, R. P.; Nikoobakht, B.; El-Sayed, M. A. Surface Reconstruction of the Unstable $\{110\}$ Surface in Gold Nanorods. J. Phys. Chem. B 2000, 104, 5417-5420.

51. Katz-Boon, H.; Walsh, M.; Dwyer, C.; Mulvaney, P.; Funston, A. M.; Etheridge, J. Stability of Crystal Facets in Gold Nanorods. Nano Lett. 2015, 15, 1635-41.

52. Gou, L. F.; Murphy, C. J. Fine-Tuning the Shape of Gold Nanorods. Chem. Mater. 2005, 17, 3668-3672.

53. Song, J. H.; Kim, F.; Kim, D.; Yang, P. D. Crystal Overgrowth on Gold Nanorods: Tuning the Shape, Facet, Aspect Ratio, and Composition of the Nanorods. Chem.-Eur. J. 2005, 11, 910-916.

54. Kou, X. S.; Zhang, S. Z.; Yang, Z.; Tsung, C. K.; Stucky, G. D.; Sun, L. D.; Wang, J. F.; Yan, C. H. Glutathione- and Cysteine-Induced Transverse Overgrowth on Gold Nanorods. J. Am. Chem. Soc. 2007, 129, 6402-6404.

55. Khlebtsov, B. N.; Ithanadeev, V. A.; Ye, J.; Sukhorukov, G. B.; Khlebtsov, N. G. Overgrowth of Gold Nanorods by Using a Binary Surfactant Mixture. Langmuir 2014, 30, 1696-1703.

56. Liu, W. Q.; Zhang, H.; Wen, T.; Yan, J.; Hou, S.; Shi, X. W.; Hu, Z. J.; Ji, Y. L.; Wu, X. C. Activation of Oxygen-Mediating Pathway Using Copper Ions: Fine-Tuning of Growth Kinetics in Gold Nanorod Overgrowth. Langmuir 2014, 30, 12376-12383.

57. Wen, T.; Zhang, H.; Tang, X. P.; Chu, W. G.; Liu, W. Q.; Ji, Y. L.; Hu, Z. J.; Hou, S.; Hu, X. N.; Wu, X. C. Copper Ion Assisted Reshaping and Etching of Gold Nanorods: Mechanism Studies and Applications. J. Phys. Chem. C 2013, 117, 25769-25777. 
58. Tsung, C. K.; Kou, X. S.; Shi, Q. H.; Zhang, J. P.; Yeung, M. H.; Wang, J. F.; Stucky, G. D. Selective Shortening of Single-Crystalline Gold Nanorods by Mild Oxidation. J. Am. Chem. Soc. 2006, $128,5352-5353$.

59. Ye, X. C.; Zheng, C.; Chen, J.; Gao, Y. Z.; Murray, C. B. Using Binary Surfactant Mixtures To Simultaneously Improve the Dimensional Tunability and Monodispersity in the Seeded Growth of Gold Nanorods. Nano Lett. 2013, 13, 765-771.

60. Ye, X. C.; Gao, Y. Z.; Chen, J.; Reifsnyder, D. C.; Zheng, C.; Murray, C. B. Seeded Growth of Monodisperse Gold Nanorods Using Bromide-Free Surfactant Mixtures. Nano Lett. 2013, 13, 21632171.

61. Ming, T.; Feng, W.; Tang, Q.; Wang, F.; Sun, L. D.; Wang, J. F.; Yan, C. H. Growth of Tetrahexahedral Gold Nanocrystals with High-index Facets. J. Am. Chem. Soc. 2009, 131, 1635016351.

62. Zhang, Q. F.; Wang, H. Facet-Dependent Catalytic Activities of Au Nanoparticles Enclosed by High-Index Facets. ACS Catal. 2014, 4, 4027-4033.

63. Tian, N.; Zhou, Z. Y.; Sun, S. G.; Ding, Y.; Wang, Z. L. Synthesis of Tetrahexahedral Platinum Nanocrystals with High-Index Facets and High Electro-Oxidation Activity. Science 2007, 316, 732735.

64. Quan, Z. W.; Wang, Y. X.; Fang, J. Y. High-Index Faceted Noble Metal Nanocrystals. Acc. Chem. Res. 2013, 46, 191-202.

65. Zhang, Q. F.; Zhou, Y. D.; Villarreal, E.; Lin, Y.; Zou, S. L.; Wang, H. Faceted Gold Nanorods: Nanocuboids, Convex Nanocuboids, and Concave Nanocuboids. Nano Lett. 2015, 15, 41614169.

66. Xia, X. H.; Zeng, J.; McDearmon, B.; Zheng, Y. Q.; Li, Q. G.; Xia, Y. N. Silver Nanocrystals with Concave Surfaces and Their Optical and Surface-Enhanced Raman Scattering Properties. Angew. Chem. Int. Edit. 2011, 50, 12542-12546. 
67. Zhang, J. A.; Langille, M. R.; Personick, M. L.; Zhang, K.; Li, S. Y.; Mirkin, C. A. Concave Cubic Gold Nanocrystals with High-Index Facets. J. Am. Chem. Soc. 2010, 132, 14012-14014.

68. Tran, T. T.; Lu, X. M. Synergistic Effect of Ag and Pd Ions on Shape-Selective Growth of Polyhedral Au Nanocrystals with High-Index Facets. J. Phys. Chem. C 2011, 115, 3638-3645.

69. Ma, Y. Y.; Kuang, Q.; Jiang, Z. Y.; Xie, Z. X.; Huang, R. B.; Zheng, L. S. Synthesis of Trisoctahedral Gold Nanocrystals with Exposed High-Index Facets by a Facile Chemical Method. Angew. Chem. Int. Edit. 2008, 47, 8901-8904.

70. Niu, W. X.; Zhang, W. Q.; Firdoz, S.; Lu, X. M. Controlled Synthesis of Palladium Concave Nanocubes with Sub-10-Nanometer Edges and Corners for Tunable Plasmonic Property. Chem. Mater. 2014, 26, 2180-2186.

71. O'Brien, M. N.; Jones, M. R.; Brown, K. A.; Mirkin, C. A. Universal Noble Metal Nanoparticle Seeds Realized Through Iterative Reductive Growth and Oxidative Dissolution Reactions. J. Am. Chem. Soc. 2014, 136, 7603-7606.

72. Lu, C. L.; Prasad, K. S.; Wu, H. L.; Ho, J. A. A.; Huang, M. H. Au Nanocube-Directed Fabrication of Au-Pd Core-Shell Nanocrystals with Tetrahexahedral, Concave Octahedral, and Octahedral Structures and Their Electrocatalytic Activity. J. Am. Chem. Soc. 2010, 132, 14546-14553.

73. Lin, H. X.; Lei, Z. C.; Jiang, Z. Y.; Hou, C. P.; Liu, D. Y.; Xu, M. M.; Tian, Z. Q.; Xie, Z. X. Supersaturation-Dependent Surface Structure Evolution: From Ionic, Molecular to Metallic Micro/Nanocrystals. J. Am. Chem. Soc. 2013, 135, 9311-9314.

74. Gou, L. F.; Murphy, C. J. Solution-phase synthesis of $\mathrm{Cu}_{2} \mathrm{O}$ nanocubes. Nano Lett. 2003, 3, 231-234.

75. Brenner, A. J.; Harris, E. D. A Quantitative Test for Copper Using Bicinchoninic Acid. Anal. Biochem. 1995, 226, 80-84.

76. Park, S.; Sinha, N.; Hamad-Schifferli, K. Effective Size and Zeta Potential of Nanorods by Ferguson Analysis. Langmuir 2010, 26, 13071-13075. 
77. Personick, M. L.; Langille, M. R.; Zhang, J.; Mirkin, C. A. Shape Control of Gold Nanoparticles by Silver Underpotential Deposition. Nano Lett. 2011, 11, 3394-3398.

78. Zhang, L.; Zhang, J. W.; Kuang, Q.; Xie, S. F.; Jiang, Z. Y.; Xie, Z. X.; Zheng, L. S. $\mathrm{Cu}^{2+}-$ Assisted Synthesis of Hexoctahedral Au-Pd Alloy Nanocrystals with High-Index Facets. J. Am. Chem. Soc. 2011, 133, 17114-17117.

79. Zhang, L.; Chen, Q. L.; Jiang, Z. Y.; Xie, Z. X.; Zheng, L. S. $\mathrm{Cu}^{2+}$ Underpotential-Deposition Assisted Synthesis of $\mathrm{Au}$ and Au-Pd Alloy Nanocrystals with Systematic Shape Evolution. CrystEngComm 2015, 17, 5556-5561.

80. Wen, T.; Hu, Z. J.; Liu, W. Q.; Zhang, H.; Hou, S.; Hu, X. N.; Wu, X. C. Copper-Ion-Assisted Growth of Gold Nanorods in Seed-Mediated Growth: Significant Narrowing of Size Distribution via Tailoring Reactivity of Seeds. Langmuir 2012, 28, 17517-17523.

81. Yang, Y.; Liu, J. Y.; Fu, Z. W.; Qin, D. Galvanic Replacement-Free Deposition of Au on Ag for Core-Shell Nanocubes with Enhanced Chemical Stability and SERS Activity. J. Am. Chem. Soc. 2014, $136,8153-8156$.

82. Raveendran, P.; Fu, J.; Wallen, S. L. A Simple and "Green" Method for the Synthesis of Au, Ag, and Au-Ag Alloy Nanoparticles. Green Chem. 2006, 8, 34-38.

83. Bai, T. L.; Sun, J. F.; Che, R. C.; Xu, L. N.; Yin, C. Y.; Guo, Z. R.; Gu, N. Controllable Preparation of Core-Shell Au-Ag Nanoshuttles with Improved Refractive Index Sensitivity and SERS Activity. ACS Appl. Mater. Interfaces 2014, 6, 3331-3340.

84. Park, K.; Vaia, R. A. Synthesis of Complex Au/Ag Nanorods by Controlled Overgrowth. $A d v$. Mater. 2008, 20, 3882-3886.

85. Jing, H.; Zhang, Q. F.; Large, N.; Yu, C. M.; Blom, D. A.; Nordlander, P.; Wang, H. Tunable Plasmonic Nanoparticles with Catalytically Active High-Index Facets. Nano Lett. 2014, 14, 36743682. 
86. Zhang, Q. F.; Blom, D. A.; Wang, H. Nanoporosity-Enhanced Catalysis on Subwavelength Au Nanoparticles: a Plasmon-Enhanced Spectroscopic Study. Chem. Mater. 2014, 26, 5131-5142.

87. Huang, J. F.; Zhu, Y. H.; Lin, M.; Wang, Q. X.; Zhao, L.; Yang, Y.; Yao, K. X.; Han, Y. SiteSpecific. Growth of Au-Pd Alloy Horns on Au Nanorods: A Platform for Highly Sensitive Monitoring of Catalytic Reactions by Surface Enhancement Raman Spectroscopy. J. Am. Chem. Soc. 2013, 135, $8552-8561$.

88. Heck, K. N.; Janesko, B. G.; Scuseria, G. E.; Halas, N. J.; Wong, M. S. Observing MetalCatalyzed Chemical Reactions in situ Using Surface-Enhanced Raman Spectroscopy on Pd-Au Nanoshells. J. Am. Chem. Soc. 2008, 130, 16592-16600.

89. Joseph, V.; Engelbrekt, C.; Zhang, J. D.; Gernert, U.; Ulstrup, J.; Kneipp, J. Characterizing the Kinetics of Nanoparticle-Catalyzed Reactions by Surface-Enhanced Raman Scattering. Angew. Chem. Int. Edit. 2012, 51, 7592-7596.

90. Xie, W.; Herrmann, C.; Kompe, K.; Haase, M.; Schlucker, S. Synthesis of Bifunctional $\mathrm{Au} / \mathrm{Pt} / \mathrm{Au}$ Core/Shell Nanoraspberries for in situ SERS Monitoring of Platinum-Catalyzed Reactions. J. Am. Chem. Soc. 2011, 133, 19302-19305.

91. Xie, W.; Walkenfort, B.; Schlucker, S. Label-Free SERS Monitoring of Chemical Reactions Catalyzed by Small Gold Nanoparticles Using 3D Plasmonic Superstructures. J. Am. Chem. Soc. 2013, $135,1657-1660$.

92. Liu, R.; Liu, J. F.; Zhang, Z. M.; Zhang, L. Q.; Sun, J. F.; Sun, M. T.; Jiang, G. B. Submonolayer-Pt-Coated Ultrathin Au Nanowires and Their Self-Organized Nanoporous Film: SERS and Catalysis Active Substrates for Operando SERS Monitoring of Catalytic Reactions. J. Phys. Chem. Lett. 2014, 5, 969-975.

93. Cui, Q. L.; Yashchenok, A.; Zhang, L.; Li, L. D.; Masic, A.; Wienskol, G.; Mohwald, H.; Bargheer, M. Fabrication of Bifunctional Gold/Gelatin Hybrid Nanocomposites and Their Application. ACS Appl. Mater. Interfaces 2014, 6, 1999-2002. 
94. DeVetter, B. M.; Mukherjee, P.; Murphy, C. J.; Bhargava, R. Measuring Binding Kinetics of Aromatic Thiolated Molecules with Nanoparticles via Surface-Enhanced Raman Spectroscopy. Nanoscale 2015, 7, 8766-8775.

95. Wang, H.; Levin, C. S.; Halas, N. J. Nanosphere Arrays with Controlled Sub-10-nm Gaps as Surface-Enhanced Raman Spectroscopy Substrates. J. Am. Chem. Soc. 2005, 127, 14992-14993.

96. Goksu, H.; Ho, S. F.; Metin, O.; Korkmaz, K.; Garcia, A. M.; Gultekin, M. S.; Sun, S. H. Tandem Dehydrogenation of Ammonia Borane and Hydrogenation of Nitro/Nitrile Compounds Catalyzed by Graphene-Supported NiPd Alloy Nanoparticles. ACS Catal. 2014, 4, 1777-1782.

97. Sun, M. T.; Xu, H. X. A Novel Application of Plasmonics: Plasmon-Driven Surface-Catalyzed Reactions. Small 2012, 8, 2777-2786.

98. Huang, Y. F.; Zhu, H. P.; Liu, G. K.; Wu, D. Y.; Ren, B.; Tian, Z. Q. When the Signal Is Not from the Original Molecule To Be Detected: Chemical Transformation of para-Aminothiophenol on Ag during the SERS Measurement. J. Am. Chem. Soc. 2010, 132, 9244-9246.

99. Hvolbaek, B.; Janssens, T. V. W.; Clausen, B. S.; Falsig, H.; Christensen, C. H.; Norskov, J. K. Catalytic Activity of Au Nanoparticles. Nano Today 2007, 2, 14-18.

100. Janssens, T. V. W.; Clausen, B. S.; Hvolbaek, B.; Falsig, H.; Christensen, C. H.; Bligaard, T.; Norskov, J. K. Insights into the Reactivity of Supported Au Nanoparticles: Combining Theory and Experiments. Top. Catal. 2007, 44, 15-26. 


\section{TOC Graphic:}

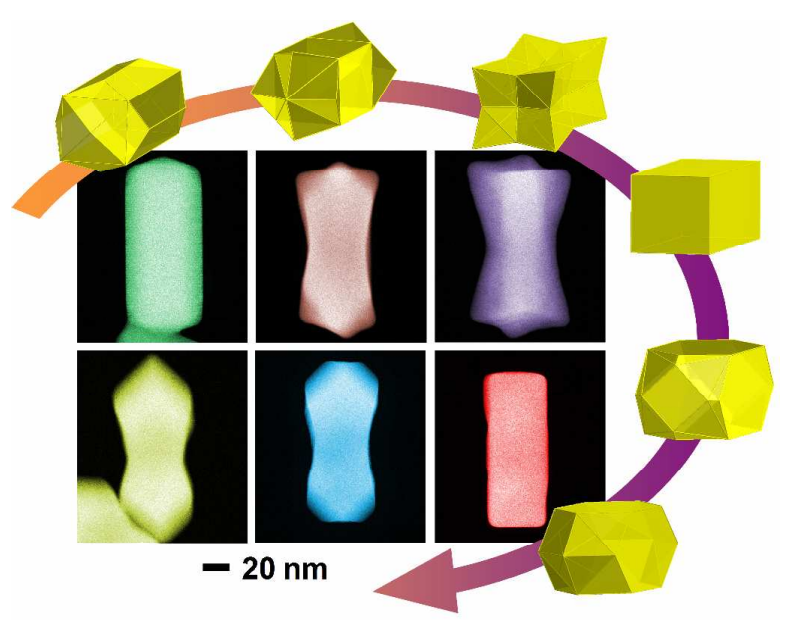

